\title{
FROM SIGNAL TRANSDUCTION TO SPATIAL PATTERN FORMATION IN E. COLI: A PARADIGM FOR MULTISCALE MODELING IN BIOLOGY*
}

\author{
RADEK ERBAN ${ }^{\dagger}$ AND HANS G. OTHMER ${ }^{\ddagger}$
}

\begin{abstract}
The collective behavior of bacterial populations provides an example of how cell-level decision making translates into population-level behavior and illustrates clearly the difficult multiscale mathematical problem of incorporating individual-level behavior into population-level models. Here we focus on the flagellated bacterium E. coli, for which a great deal is known about signal detection, transduction, and cell-level swimming behavior. We review the biological background on individual- and population-level processes and discuss the velocity-jump approach used for describing population-level behavior based on individual-level intracellular processes. In particular, we generalize the moment-based approach to macroscopic equations used earlier [R. Erban and H. G. Othmer, SIAM J. Appl. Math., 65 (2004), pp. 361-391] to higher dimensions and show how aspects of the signal transduction and response enter into the macroscopic equations. We also discuss computational issues surrounding the bacterial pattern formation problem and technical issues involved in the derivation of macroscopic equations.
\end{abstract}

Key words. aggregation, chemotaxis equations, pattern formation, velocity-jump processes

AMS subject classifications. 35Q80, 92B05

DOI. $10.1137 / 040603565$

1. Introduction. Appropriate responses to signals in the environment are a sine qua non for survival of any organism, and thus sophisticated means of detecting external signals, transducing them into internal signals, and altering behavioral patterns appropriately have evolved. Many organisms use a random walk search strategy to search for food when the signals are spatially uniform and bias movement appropriately when a suitable change in signal is detected. A well-studied example of this is the motion of flagellated bacteria such as $E$. coli, which serves as the primary example in this paper. However, the methods presented here can be applied to any random walker after suitable modification of the underlying biological details.

One of the ways that motile systems may respond to environmental signals is by changing their speed or the frequency of turning, a process called kinesis. Chemokinesis, which involves changes in speed or turning frequency in response to chemicals, has been studied most in E. coli, which has $4-6$ helical flagella and swims by rotating them $[66,55]$. When rotated counterclockwise (CCW) the flagella coalesce into a propulsive bundle and lead to a "run" [9], but when rotated clockwise (CW) the bundle dissociates and the cell tumbles in place, thereby generating a random direction for the next run. A stochastic process generates the runs and tumbles, and in an attractant gradient runs that carry the cell in a favorable direction are extended. The cell senses

* Received by the editors January 26, 2004; accepted for publication (in revised form) June 7, 2004; published electronically February 9, 2005.

http://www.siam.org/journals/mms/3-2/60356.html

†School of Mathematics, 270B Vincent Hall, University of Minnesota, Minneapolis, MN 55455 (erban@math.umn.edu). This author's research was supported in part by NSF grant DMS 0317372.

${ }^{\ddagger}$ Max Planck Institute for Mathematics in the Sciences, 22 Inselstrasse, Leipzig, Germany D04103. Current address: School of Mathematics, 270A Vincent Hall, University of Minnesota, Minneapolis, MN 55455 (othmer@math.umn.edu). This author's research was supported in part by NIH grant GM 29123, NSF grants DMS 9805494 and DMS 0317372, the Max Planck Institute, Leipzig, and the Alexander von Humboldt Foundation. 
spatial gradients as temporal changes in receptor occupancy and changes the probability of CCW rotation (the bias) on a fast time scale. Adaptation returns the bias to baseline on a slow time scale, enabling the cell to detect and respond to further concentration changes. The motion of E. coli can be characterized as a velocity-jump process [46] because an individual runs in given direction, but at random instants of time it stops to choose a new velocity, and the time spent in the latter stage is small compared to the run length. As we will see later, this description provides the starting point for deriving macroscopic equations that incorporate microscopic behavior.

$E$. coli has five receptor types which communicate with the flagellar motors via a biochemical pathway (described in more detail later) that ends in the motor control protein CheY [22]. CCW is the default state in the absence of the phosphorylated CheY, which binds to motor proteins and increases CW rotation. Attractant binding to a receptor reduces the phosphorylation rate of $\mathrm{CheY}$ and thereby increases the bias, which constitutes the fast response to a signal. Adaptation involves changes in the methylation state of the receptor, which is set by the balance between methylation of sites by the methyltransferase CheR and demethylation by the methylesterase CheB. These key steps, excitation via reduction in $\mathrm{CheY}_{\mathrm{p}}$ when a receptor is occupied and adaptation via methylation of the receptors, have been incorporated in mathematical models of signal transduction [64, 3, 45], but important aspects of signal transduction are still not understood. For example, E. coli can sense and adapt to ligand concentrations spanning five orders of magnitude and can detect a change in occupancy of the aspartate receptor Tar of as little as 0.1\% [10]. The gain of the system, defined as the change in bias divided by the change in receptor occupancy, can be as high as 55 [58], and a long-standing question is where in the pathway from ligand to motor this high gain resides. Thus the major characteristics of individual behavior that must be captured by models are (i) fast changes in the bias in response to a change in signal, (ii) slow adaption to maintained stimuli, and (iii) high gain or sensitivity.

In addition to the complex individual behavior just described, bacterial populations exhibit various collective behaviors, including spatial pattern formation, quorum sensing, and formation of biofilms. Of course these involve individual-level responses to signals, but they arise in populations that may comprise millions of individual bacteria, which raises the question of how to incorporate the individual behavioral rules into the population-level models. As shown in Table 1.1, the relevant physical processes occur over a vast range of time scales, and thus $E$. coli is a perfect paradigm of multiscale modeling in biology. Usually, microscopic aspects of individual behavior are incorporated into macroscopic descriptions phenomenologically, but recently significant progress on closing the gap between micro- and macroscopic models has been made, and how this is done is described later. A start on this was made in [21], where the one-dimensional problem of bacterial chemotaxis was solved for a simple model that describes some essential behavioral aspects of $E$. coli, and here we extend this approach to higher dimensions.

In section 2 we review the cell-level processes involved in signal transduction, motor control, and pattern formation in E. coli. Since some readers may wish to skip these details, the paper is written so that this section can be omitted on a first reading. In section 3, we discuss a cartoon description that captures much of the essential behavior of the detailed signal transduction network and then describe the theoretical methods that have been used to address the problems encountered in trying to lift the cell-level behavior to a population-level description. Next we provide the generalization of the one-dimensional method from [21] to higher dimensions. The method in [21] cannot be used directly and must be modified in more than one space 
TABLE 1.1

Examples of the time scales of basic processes in E. coli.

\begin{tabular}{|c|c|c|}
\hline Characteristic time & Process & Reference(s) \\
\hline $0.01 \mathrm{sec}$ & ligand binding, receptor phosphorylation & {$[12],[71]$} \\
$0.1 \mathrm{sec}$ & mean tumbling time & {$[8]$} \\
$1 \mathrm{sec}$ & mean running time & {$[8]$} \\
several seconds & adaptation time, receptor methylation & {$[62],[71]$} \\
hour(s) & proliferation & {$[30]$} \\
$2-3$ days & pattern formation experiments & {$[15],[16]$} \\
\hline
\end{tabular}

dimension. We use two methods to treat this problem, both of which lead to the multidimensional counterparts of equations derived in [21]. In section 4 , we derive a macroscopic equation (4.40) using a very general setup, and we derive the classical chemotaxis equation (4.44) for bacterial movement by specializing the general theory. In section 5, we discuss another approach to deriving multidimensional macroscopic equations, which leads to hyperbolic models for chemotaxis. In section 6 , we show some illustrative numerical results. Finally, we discuss the possible extension of the methods to modeling of bacterial pattern formation and discuss new computational methods that may prove useful in this context in section 7 .

2. The biochemical and biophysical aspects of individual and collective behavior in $\boldsymbol{E}$. coli. Understanding how complex networks produce the desired output in response to signals and how that output is buffered against the inevitable fluctuations in the molecular levels of the components is a major problem in biology, whether in the context of gene control networks in development or at the level of cells or organisms. Chemotaxis in E. coli provides an excellent model system for understanding how reliable cell-level behavior emerges from a complex signal transduction and control network, in large part because all the major components are known, yet there are fundamental issues that remain to be understood. In this section we describe the cell-level processes involved in signal transduction and motor control and discuss pattern formation in populations of E. coli.

2.1. Signal transduction and adaptation. As was described earlier, E. coli alternates two basic behavioral modes based on CCW and CW flagellar rotation to search for food or escape an unfavorable environment. CCW rotation pushes the cell forward in a straight "run" with a speed $s=10-20 \mu \mathrm{m} / \mathrm{sec}$, and CW rotation of flagella triggers a random "tumble" that reorients the cell. In the absence of an extracellular signal the duration of both runs and tumbles are exponentially distributed, with means of $1 \mathrm{~s}$ and $10^{-1} \mathrm{~s}$, respectively, and in a gradient of attractant the cell increases or decreases the run time depending on whether it moves in a favorable or unfavorable direction. During a run the bacteria move at approximately constant speed in the most recently chosen direction, and new directions are generated during tumbles. The distribution of new directions is not perfectly uniform on the unit sphere but has a slight bias in the direction of the preceding run.

This mechanism by which bacteria move in favorable directions is often called chemotaxis, but it is more precisely called chemokinesis since it involves changes in the frequency of turning, not in the direction of movement. However, the term chemotaxis is so widely used in the context of bacteria that we adopt it here to describe the process by which a cell alters its movement in response to an extracellular chemical signal. A schematic of the signal transduction pathway is shown in Figure 2.1(a). The key 


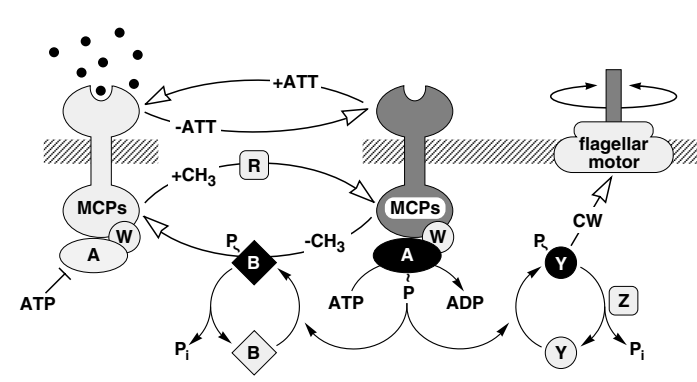

(a)

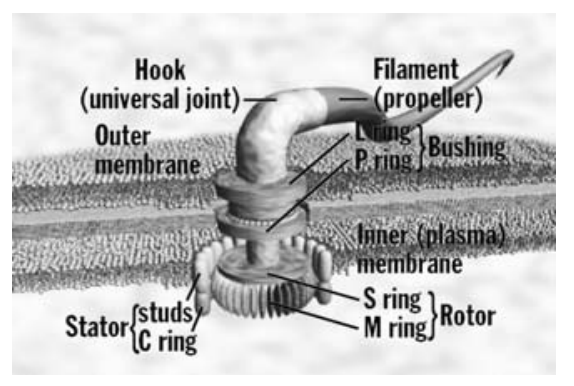

(b)

FIG. 2.1. (a) The signal transduction pathway in E. coli. Chemoreceptors (MCPs) span the cytoplasmic membrane (hatched lines), with a ligand-binding domain on the periplasmic side and a signaling domain on the cytoplasmic side. The cytoplasmic signaling proteins, denoted Che in the text, are identified by single letters, e.g., $A=$ CheA. (From [64], with permission. (C) 1997 National Academy of Sciences, U.S.A.) (b) A detail of the motor. (From Access Research Network (http://www.arn.org/docs/mm), with permission.)

aspects of behavior that must be explained by a model are as follows.

(1) The response of a tethered cell to a small step change in the signal in a spatially uniform environment occurs within 2-4 seconds [9]. This is also approximately the mean period during which cell motion persists up a positive spatial gradient of attractant and is thus the time scale over which changes in concentration during movement are measured [9]. This response time is considered optimal, because statistical fluctuations make measurements over shorter time scales less accurate $[7,6]$. Large changes in chemoeffector concentration, which saturate the cell's chemoreceptors, can increase the response time to several minutes [67]. As in many other sensory systems, the signal transduction pathway in E. coli adapts to constant stimuli (cf. Figure 2.2(a)), by which we mean that after a change in signal the cell's response, defined as a change in the bias from its baseline value, eventually returns to zero. As a result, the signal transduction system can respond to stimuli ranging over $4-5$ orders of magnitude [10].

(2) E. coli is sensitive to small changes in chemoeffector levels: cells can respond to slow exponential increases in attractant levels that correspond to rates of change in the fractional occupancy of chemoreceptors as small as $0.1 \%$ per second $[8,58]$. High sensitivity is also seen when cells are subjected to small impulses or step increases in attractant concentration. The gain of the system, defined as the change in bias divided by the change in receptor occupancy, can be as high as 55 [58], and a long-standing question is where in the pathway from ligand to motor this high gain resides. One source is apparent cooperative binding of $\mathrm{CheY}_{\mathrm{p}}$ to the motor proteins. The analysis done in Spiro, Parkinson, and Othmer [64] showed that in the absence of cooperativity in signal transduction, a Hill coefficient of at least 11 for binding of $\mathrm{CheY}_{\mathrm{p}}$ at the motor was needed to explain the observed gains of 3-6 [64], and recent experiments have confirmed this prediction [18] (cf. Figure 2.2(b)). However, this does not account for all of the highest observed gains, and it has recently been shown that the stage between aspartate binding and $\mathrm{CheY}_{\mathrm{p}}$ concentration has an amplification 35 times greater than expected [63]. None of the existing models of the full signal transduction system incorporate this source of gain $[64,3,45]$, but a model that describes the initial stage of transduction has been developed [1]. It remains to incorporate this into a model for the entire pathway.

Some of the details involved in signal transduction are as follows. Extracellular 


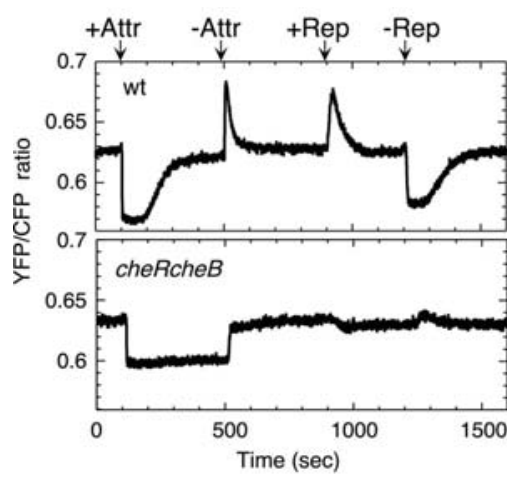

(a)

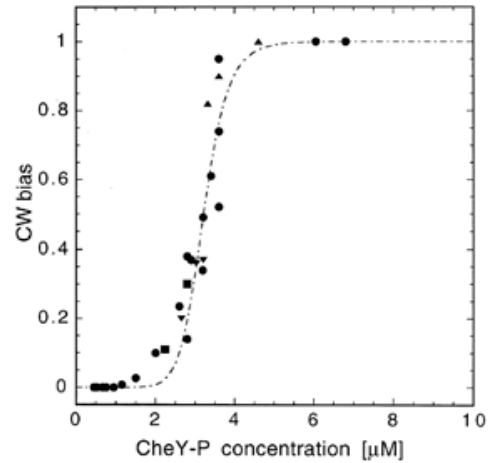

(b)

FIG. 2.2. (a) The measured response of Che $Y_{p}$ to the addition and removal of attractant or repellent for wild-type cells (upper) and the response of a mutant that lacks two key enzymes in the signal transduction pathway (lower). (From [63], with permission. (C) 2002 National Academy of Sciences, U.S.A.) (b) The clockwise bias of a single flagellum as a function of the Che $Y_{p}$ concentration. (Reprinted with permission from [18]. (C) 2000 AAAS.) The curve can be fit with a Hill function of the form $R(Y)=Y^{H} /\left(K^{H}+Y^{H}\right)$, where $R(Y)$ is the response, $Y$ is the Che $Y_{p}$ concentration, and $K$ is the half-maximal concentration. The estimated Hill coefficient is $H=$ $10.2 \pm 1$.

signals are detected by transmembrane receptors, which in turn generate intracellular signals that control the flagellar motors (see Figure 2.1(a)). Aspartate, the attractant most commonly used in experiments, binds directly to the periplasmic domain of its receptor, Tar. The cytoplasmic domain of Tar forms a stable complex with the signaling proteins CheA and CheW, and the stability of this complex is not affected by ligand binding [27]. The signaling currency is in the form of phosphoryl groups $(\sim \mathrm{P})$, made available to the $\mathrm{CheY}$ and $\mathrm{CheB}$ proteins through autophosphorylation of CheA. $\mathrm{CheY}_{\mathrm{p}}$ initiates flagellar responses by interacting with the motor to enhance the probability of $\mathrm{CW}$ rotation. $\mathrm{CheB}_{\mathrm{p}}$ is part of a sensory adaptation circuit that terminates motor responses. MCP complexes have two alternative signaling states. In the attractant-bound form, the receptor inhibits CheA autokinase activity; in the unliganded form, the receptor stimulates CheA activity. The overall flux of phosphoryl groups to $\mathrm{CheB}$ and $\mathrm{CheY}$ reflects the proportion of signaling complexes in the inhibited and stimulated states. Changes in attractant concentration shift this distribution, triggering a flagellar response. The ensuing changes in CheB phosphorylation state alter its methylesterase activity, producing a net change in MCP methylation state that cancels the stimulus signal (see Stock and Surette [68] for a review).

Several detailed mathematical models for signal transduction and adaptation have been developed [64, 3, 45]. The model in Spiro, Parkinson, and Othmer [64] is based on the assumption that Tar is the only receptor type, that the Tar-CheA-CheW complex does not dissociate, and that Tar, CheA, and CheW are found only in this complex. The primary objective is to model the response to attractant, which probably involves only increases in the average methylation level above the unstimulated level of about 1.5-2 methyl esters per receptor [11], and for this reason the model incorporates only the three highest methylation states of Tar, and only the phosphorylated form of CheB $\left(\mathrm{CheB}_{\mathrm{p}}\right)$ has demethylation activity. In Figure 2.3(a) we illustrate the various states of the receptor complex and the transitions between them. We use $T$ to represent the concentration of Tar-CheA-CheW complex and $L$ to represent ligand concentration. 


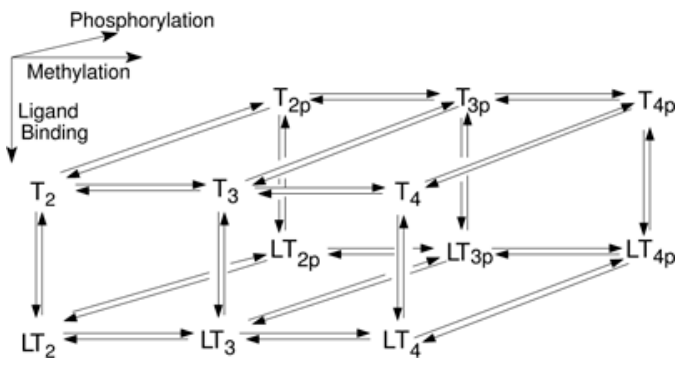

(a)

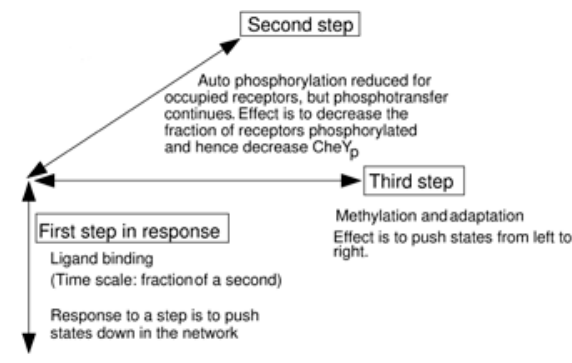

(b)

FIG. 2.3. (a) Illustration of the ligand-binding, phosphorylation, and methylation reactions undergone by the Tar-CheA-CheW complex (denoted by T) in the full model. (From [64], with permission. (C) 1997 National Academy of Sciences, U.S.A.) (b) A schematic that shows the three primary processes and their temporal ordering. Of course the processes overlap in time, but for explanatory purposes we separate them.

A $p$ subscript indicates a phosphorylated species, and numerical subscripts indicate the number of methylated sites on Tar. The details of all assumptions underlying the model and the kinetics of the reactions in this figure are given in Spiro, Parkinson, and Othmer [64].

A qualitative description of how the system works is as follows (cf. Figure 2.3(b)). The ligand-binding reactions are the fastest, and thus a step increase in attractant first shifts the equilibrium among the receptor states in Figure 2.3(a) toward the ligandbound states at the bottom face of the box. This increases the fraction of receptors in the sequestered states $\left(L T_{2}\right.$, and possibly $\left.L T_{3}\right)$. These states have much lower autophosphorylation rates than the corresponding unliganded states, and therefore the equilibrium next shifts toward states containing unphosphorylated CheA, which are in the front face of the box. The level of phosphorylated CheA $\left(\mathrm{CheA}_{\mathrm{p}}\right)$ is thus lowered, causing less phosphate to be transferred to CheY, yielding a lowered level of $\mathrm{CheY}_{\mathrm{p}}$. As a result, tumbling is suppressed, and the cell's run length increases. This constitutes the excitation response of the system.

Next, the methylation and demethylation reactions - the slowest reactions in this system-begin to influence the response. Ligand-bound receptors are more readily methylated than unliganded receptors, and the lowered level of $\mathrm{Che}_{\mathrm{p}}$ causes a decrease in the level of $\mathrm{CheB}_{\mathrm{p}}$, thereby reducing its demethylation activity. As a result, the equilibrium shifts in the direction of the higher methylation states, toward the right face of the box. The autophosphorylation rate of CheA is faster when the associated Tar-CheA-CheW complex is in a higher methylation state, and so there is finally a shift back toward the states containing $\mathrm{CheA}_{\mathrm{p}}$, at the rear face of the box. As a result, $\mathrm{CheY}_{\mathrm{p}}$ returns to its prestimulus level, and thus so does the bias of the cell. This constitutes the adaptation response. The net effect of the increase in attractant is thus to shift the distribution of receptor states toward those which are ligand-bound and more highly methylated, but the total level of receptor-complex containing $\mathrm{CheA}_{\mathrm{p}}$ (the sum of the states at the rear face of the box) returns to baseline, leaving the cell capable of responding to a further change in ligand concentration. In order to obtain perfect adaptation, the level of phosphorylated receptor must return to its prestimulus level, and this occurs because CheA autophosphorylates more when the receptor is more highly methylated.

The mathematical description of the model is based on mass action kinetics and 
singular perturbation of certain steps [64]. Numerical solution of the equations in [64] shows that the model can capture most aspects of the stimulus-response behavior, including the response to small and large steps and to slow ramps. At present it is not understood how such detailed microscopic models can be incorporated into macroscopic models, but the essential aspects of the dynamics can be captured by a much simpler "cartoon" model that will be described in section 3.3.1.

2.2. Motor control. The effect of changes in $\mathrm{CheY}_{\mathrm{p}}$ is to change the bias of the motor, and thus another component of a model is to describe the interaction of $\mathrm{CheY}_{\mathrm{p}}$ with the motor proteins at the flagellar switch [17]. At present it is estimated that there are about 30 binding sites for $\mathrm{CheY}_{\mathrm{p}}$ on the motor, and hence 30 equations describing their occupancy states, but singular perturbation techniques can reduce this to a single equation describing the switching of the motor between the two rotational states. The kinetic rates for these transitions depend on $\mathrm{CheY}_{\mathrm{p}}$ and can be estimated from data in [18]. Finally, a cell has a much higher bias than an individual flagellum, and in Spiro, Parkinson, and Othmer [64] it is shown that this can be understood by invoking the "voting hypothesis" for the collective behavior of the flagella. Details on how this is implemented are given in [60].

2.3. Pattern formation. It is relatively recently that the importance of multicellular organization in prokaryotes, aside from examples such as Myxoccus xanthus, has been recognized [61]. It is now known that intercellular communication and coordination are widespread in prokaryotes, and many different classes of signaling molecules have been identified. Current interest in this phenomenon stems from the discovery of quorum-sensing molecules such as the N-acyl homoserine lactones and their possible role in biofilm formation [23]. A connection with the behaviors described here comes from evidence that the initial microcolonies may be formed by aggregation of bacteria and that active motility plays a role in the formation of biofilms because it can counter repulsive forces at the surface being colonized and thus foster successful attachment. Much simpler, and more tractable from the modeling standpoint, examples of pattern formation arise in E. coli, S. typhimurium, and B. subtilis colonies [15, 16, 61] (cf. Figure 2.4). Pattern formation in E. coli, which we focus on here, involves sensing of extracellular signals and alterations in the swimming behavior of individuals, as well as production of the attractant. However, spatial patterns can involve millions of cells, and heretofore modeling of them has been primarily phenomenological; details of the cell-level behavior has not been incorporated into the equations that describe cell populations.

Experimental work has defined the conditions under which complex spatial patterns can arise $[15,5,4,16,44]$. One type of experiment is done on agar plates with agar concentration low enough that the bacteria can swim freely. Different spatial patterns can form when $E$. coli in a chemotactically inert environment are stressed by a variety of conditions, any of which is called an inducer, including introduction of components of the TCA cycle (e.g., malate, fumarate, or succinate), antibiotics, and cold shock [14]. Aggregation is in response to the production of aspartate and/or glutamate: asp $A^{-}$cells cannot produce secreted aspartate in response to inducers and do not aggregate under conditions that induce aggregation in wild-type cells [14]. In the experiments, a medium containing an inducer such as succinate is added to the agar, and cells are then introduced at one point in space. Pattern changes occur on a time scale of hours, and growth and division are essential. The doubling time is constant at about $2 \mathrm{hrs}$ under all conditions studied [14]. Much is known about the response when succinate is the inducer: cells internalize it, and it provides 


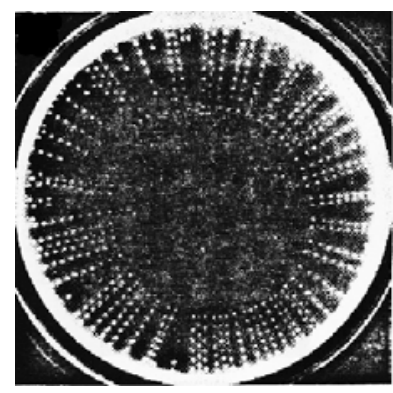

(a)

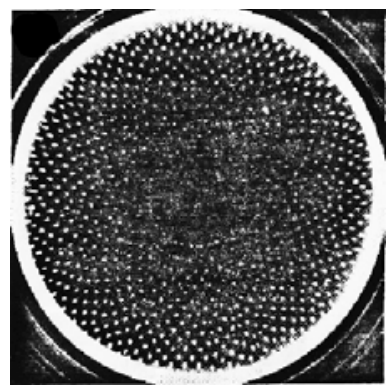

(b)

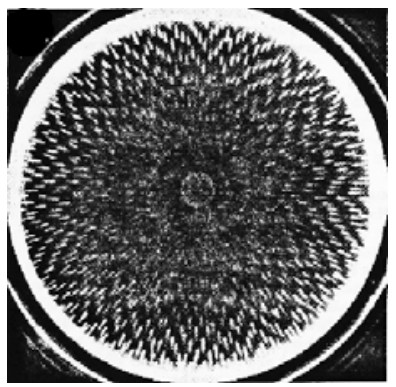

(c)

FIG. 2.4. Spatial patterns that arise in E. coli. Light denotes high cell density. (From [15], with permission. (c) 1991 Nature Publishing Group.)

precursors for certain steps in the TCA cycle that end in the production and secretion of aspartate.

The initial response in the on-agar experiments involves significant growth and division at the site of inoculation. Initially, bacteria disperse from the inoculation site, but as aspartate concentration increases they respond chemotactically and the aggregate becomes denser. As a result, succinate is depleted locally and aspartate production begins to drop. Cells at the boundary of the aggregate still receive the succinate diffusing inward and continue to produce aspartate. Thus a ring of aspartate-producing cells may develop and move radially outward. The progression of the patterns radially outward is complicated and depends on the concentration of succinate. At low succinate concentrations the ring may propagate outward without breaking up, but at higher concentrations it breaks up into spots (cf. Figure 2.4). If the concentration is not too high, the spots are aligned radially (cf. Figure 2.4(a)), at least until the radius is very large, in which case new spots appear between the old ones. The radial sequence of spots is formed by a repetition of the sequence that initiated the first ring. The local cell density in a spot grows, causing an increase in the aspartate, the local density at a spot increases further, the succinate becomes depleted locally, and the spot disintegrates; bacteria move from the spot towards the faint front and join the front, and a new spot forms above the old spot. The faint front consists of cells that are aggregating in a fluted pattern at the leading edge, and these form the basis of the next ring. At not too high succinate concentrations the cells in one ring simply migrate radially to the next ring. However, if the succinate concentration is high enough, the production of aspartate is high enough in the parabolic "chains" that form at the leading edge, and a spot forms on a ray at an angle between those of the nearest spots in the preceding "generation" (cf. Figure 2.4(b)). The speed at which the front moves is constant and inversely proportional to the succinate concentration, and the radius at which the ring collapses into spots is also inversely proportional to the succinate concentration. Both follow from the fact that at higher concentrations aspartate production is higher chemotaxis stronger. This is the experimentally observed sequence of spatio-temporal patterning that models must replicate.

Several models for pattern formation in B. subtilus and S. typhimurium based on Keller-Segel-type equations or hydrodynamic models have been proposed [4, 73, $72,36,41]$. Additional mechanisms that are incorporated include nonlinearity in the chemotactic coefficient [73], a second repellent field, or the autocatalytic production 
of attractant triggered by waste $[4,72]$. In addition to the fact that the biochemistry of signal transduction and behavioral response is different and largely unknown in these genera, there are significant differences in the pattern formation between them. Salmonella is closest to E. coli but still very different: in the former, patterns grow from an existing lawn of bacteria, while in the latter, patterns emerge from point inoculation sites, and patterns in Salmonella are less symmetrical and less structured than in E. coli. Thus, while similar processes are involved, the details are quite different in the two systems. Brenner, Levitov, and Budrene [13] model E. coli patterns and couple a Keller-Segel chemotaxis equation with equations for succinate and aspartate. They show that the movement of the swarm rings may be due to the depletion of succinate around the band of bacteria: chemotaxis holds the band together, but the net motion is not caused directly by chemotactic fluxes. In contrast, aggregates result from a purely chemotactic response in the system, depending only weakly on environmental conditions. However, their analysis is based on a phenomenological approach to chemotaxis and does not incorporate the individual response into the macroscopic equations. More recently, Mittal et al. [44] have proposed a nonmechanistic, formal model for spot formation in $E$. coli that provides insight into some of the controlling processes.

2.4. Biofilms. The patterns formed in the experiments described in the previous section are examples of rudimentary biofilms, which in general are complex structures that comprise microorganisms such as bacteria embedded in a polysaccharide matrix and attached to a surface. Biofilms are responsible for a large percentage of the infections in the body, including oral infections, gastrointestinal tract infections, and infections associated with implanted and prosthetic devices. In mixed populations bacteria may communicate with each other within a biofilm, and they can secrete molecules that signal when the population has reached a critical threshold. This process, called quorum sensing, is responsible for the expression of various factors that control synthesis of virulence factors and other substances. It is also known that there is increased antibiotic resistance in biofilm communities, which may make it difficult to combat infections and colonizations of surfaces. In addition, bacteria within biofilms may be protected from the normal immune response, in that leukocytes or phagocytes may be unable to reach them. There is also evidence that specific selective forces act in a biofilm, which lead to new cell types via facilitated gene exchange. Interested readers may consult $[2,19,54,57]$ for further details.

3. Mathematical models for bacterial chemotaxis. To date, most macroscopic models take the form of PDEs based on phenomenological assumptions relating the macroscopic cell velocity to signal gradients, etc., as described later. Such models can often explain aggregation qualitatively, but they are very restrictive quantitatively. To explain aggregation quantitatively, one must first have a detailed model of a single cell of the type described earlier, but then one must lift this to the collective behavior of the population of individuals. In this section we first show how the phenomenological route to the classical chemotaxis equations can be partially justified using a velocity-jump process with phenomenological descriptions of how the motion of individuals is biased by a signal. Then we develop the equations that incorporate intracellular dynamics and summarize the main results in one space dimension from [21]. In the remainder of the paper, we generalize the ideas from [21] to higher dimensions.

3.1. The classical phenomenological approach. In the absence of external queues, many organisms use a random walk strategy to determine their pattern of 
movement. The random walk may be in physical space and is then called a space-jump process, or it may be in velocity space and involve discontinuous changes in the speed or direction generated by a Poisson process, in which case it is called a velocity-jump process [46]. If the walkers are noninteracting, the former leads to a renewal equation in which the kernel governs the waiting time between jumps and the redistribution after a jump [46]. By suitable choices of these functions the movement of organisms released at a point in a uniform environment is described by the solution of the standard diffusion equation on a sufficiently long time scale. Thus, in an appropriate continuum limit the cell density $n$, measured in units of cells $/ \mathrm{L}^{N}$, where L denotes length and $N=1,2$, or 3 , satisfies the diffusion equation

$$
\frac{\partial n}{\partial t}=D \Delta n .
$$

The cell flux is given by $j=-D \nabla n$, and if we define the average cell velocity $u$ by the relation $j=n u$, then we see that for pure diffusive spread $u=-D \nabla n / n$.

The simplest description of cell motion in the presence of an attractant or repellent is obtained by adding a directed component to the diffusive flux to obtain

$$
j=-D \nabla n+n u_{c},
$$

where $u_{c}$ is the macroscopic chemotactic velocity. The taxis is positive or negative depending on whether $u_{c}$ is parallel or antiparallel to the direction of increase of the chemotactic substance. The resulting evolution equation is

$$
\frac{\partial n}{\partial t}=\nabla \cdot\left(D \nabla n-n u_{c}\right)
$$

and this is called a chemotaxis equation. Commonly used constitutive equations postulate that

$$
u_{c}=\chi(S) \nabla S,
$$

where $S$ is the concentration of the chemotactic substance and the function $\chi(S)$ is called the chemotactic sensitivity. When $\chi(S)>0$, the tactic component of the flux is in the direction of $\nabla S$ and the taxis is positive. With this postulate, (3.3) can be written in the form

$$
\frac{\partial n}{\partial t}=\nabla \cdot(D \nabla n-n \chi(S) \nabla S)
$$

If a diffusible chemoattractant is produced or degraded, its evolution can be described by the reaction-diffusion equation

$$
\frac{\partial S}{\partial t}=D_{S} \Delta S+r(S, n) n
$$

where $r$ is the net rate of production of the attractant per unit of cell density. We call the system of equations (3.5)-(3.6) the Keller-Segel [37, 38] or classical chemotaxis system.

Another method, based on a continuous time reinforced random walk in which the walker modifies the transition probabilities of an interval for successive crossings, is developed in [48] for a single tactic substance and in [52] for multiple substances. More recent results are given in [51,34]. 
This phenomenological approach poses the problem of connecting microscopic and macroscopic descriptions very clearly; namely, how does one justify the constitutive assumption (3.4) to describe taxis, and, in particular, how can one incorporate microscopic responses of individual cells into population-level functions such as the chemotactic sensitivity? We begin by describing a general stochastic process that can model the movement of swimming bacteria and then describe a simple example that can be worked out in detail to understand minimal properties needed in more complex models. A related approach for amoeboid chemotaxis is given in [20].

3.2. The velocity-jump process without internal dynamics. In the absence of interaction between particles or bias in their movement due to imposed external signals, the velocity-jump process leads to the transport equation

$$
\frac{\partial}{\partial t} p(x, v, t)+v \cdot \nabla p(x, v, t)=-\lambda p(x, v, t)+\lambda \int_{V} T\left(v, v^{\prime}\right) p\left(x, v^{\prime}, t\right) d v^{\prime}
$$

for the density of particles at $x \in \Omega \subset \mathbb{R}^{N}$, moving with velocity $v \in V \subset \mathbb{R}^{N}$ at time $t \geq 0$ [46]. $\lambda$ is the turning rate, and $1 / \lambda$ is a measure of the mean run time between velocity jumps. Here $\lambda$ is constant, but later it may be space-dependent and depend on internal and external variables as well. The turning kernel $T\left(v, v^{\prime}\right)$, which may also be space-dependent, gives the probability of a velocity jump from $v^{\prime}$ to $v$ if a jump occurs, and implicit in the above formulation is the assumption that the choice of a new velocity is independent of the run length. When applied to the bacterium E. coli, the turning frequency depends on the extracellular signal, as transduced through the signal transduction and motor control system.

The backward equation that corresponds to (3.7) has been derived from the underlying stochastic velocity-jump process by Stroock [69] to describe the motion of bacteria. It has also been derived and analyzed in a more general framework by Papanicolaou [53], and if we assume additional regularity of the process, one can derive (3.7) rigorously as the forward equation of the velocity-jump process. The transport equation (3.7) is similar to the Boltzmann equation, in which case the right-hand side is an integral operator that describes collision of two particles. It is known that the long time behavior of solutions of transport equations is that of a diffusion process under suitable hypotheses [40,29], and for (3.7) the diffusion tensor can be directly related to properties of the turning kernel [32]. The effect of externally imposed biases on the turning rate or the turning kernel then leads to the classical chemotaxis equation under various hypotheses [49]. To understand how internal dynamics must modulate turning behavior to produce this equation, we first analyze a simple example.

3.2.1. The telegraph process. The simplest example that illustrates how localization or aggregation of walkers depends on the parameters of the jump process is given by a one-dimensional version of (3.7) called the telegraph process. Suppose that a particle moves along the $x$-axis at a speed $s^{ \pm}(x)$ that depends on $x$ and its direction of travel, and suppose that at random instants of time it reverses direction. Let $p^{ \pm}(x, t)$ be the density of particles that are at $(x, t)$ and are moving to the right $(+)$ and left $(-)$. Then $p^{ \pm}(x, t)$ satisfy the following equations (cf. [47, 32]):

$$
\begin{aligned}
& \frac{\partial p^{+}}{\partial t}+\frac{\partial\left(s^{+} p^{+}\right)}{\partial x}=-\lambda p^{+}+\lambda p^{-} \\
& \frac{\partial p^{-}}{\partial t}-\frac{\partial\left(s^{-} p^{-}\right)}{\partial x}=\lambda p^{+}-\lambda p^{-}
\end{aligned}
$$


The density of particles at $(x, t)$ is $n(x, t) \equiv p^{+}(x, t)+p^{-}(x, t)$, and the flux is $j \equiv$ $\left(s^{+} p^{+}-s^{-} p^{-}\right)$. These satisfy the equations

$$
\begin{aligned}
\frac{\partial n}{\partial t}+\frac{\partial j}{\partial x} & =0, \\
\frac{\partial j}{\partial t}+\lambda j & =-s^{+} \frac{\partial}{\partial x}\left(s^{+} p^{+}\right)-s^{-} \frac{\partial}{\partial x}\left(s^{-} p^{-}\right)+\lambda\left(s^{+} p^{-}-s^{-} p^{+}\right)
\end{aligned}
$$

and the initial conditions $n(x, 0)=n_{0}(x), j(x, 0)=j_{0}(x)$, where $n_{0}$ and $j_{0}$ are determined from the initial distribution of $p^{+}$and $p^{-}$. When the speed and turning rate are constant, the system reduces to the telegraph equation

$$
\frac{\partial^{2} n}{\partial t^{2}}+2 \lambda \frac{\partial n}{\partial t}=s^{2} \frac{\partial^{2} n}{\partial x^{2}} .
$$

This process has been studied by many authors [70, 24, 28, 35, 43, 59, 46].

The diffusion equation results by formally taking the limit $\lambda \rightarrow \infty, s \rightarrow \infty$ with $s^{2} / \lambda \equiv 2 D$ constant in (3.10), but this can be made more precise because the equation can be solved explicitly. The fundamental solution of (3.10) can be expressed in terms of the modified Bessel functions [74], and using asymptotic properties of these functions one can show [74] that

$$
n(x, t)=\frac{1}{\sqrt{4 \pi D t}} e^{-x^{2} / 4 D t}+e^{-\lambda t} \mathcal{O}\left(\zeta^{2}\right),
$$

where $\zeta \equiv x / s t$. Therefore when $x \ll s t$ the telegraph process can be approximated by a diffusion process. If we define $\tau=\epsilon^{2} t$ and $\xi=\epsilon x$, where $\epsilon$ is a small parameter, then (3.10) reduces to

$$
\epsilon^{2} \frac{\partial^{2} n}{\partial \tau^{2}}+2 \lambda \frac{\partial n}{\partial \tau}=s^{2} \frac{\partial^{2} n}{\partial \xi^{2}}
$$

The diffusion regime defined by the exact solution now becomes

$$
\frac{x}{s t}=\epsilon \frac{\xi}{s \tau}
$$

and this requires only that $\xi /(s \tau) \leq \mathcal{O}(1)$. In the limit $\epsilon \rightarrow 0$ the exact solution can be used to show that (3.12) again reduces to the diffusion equation, both formally and rigorously (for $t$ bounded away from zero). However, this shows that the approximation of the telegraph process by a diffusion process hinges on the appropriate relation between the space and time scales, not necessarily on the limit of speed and turning rate tending to infinity, as is well known.

To illustrate how variable speeds and turning rates affect the existence of nonuniform steady states, which can be interpreted as aggregations, consider the system (3.9) on the interval $[0,1]$ and impose Neumann (no-flux) boundary conditions at both ends. We wish to know under what conditions, if any, these equations have time-independent, nonconstant solutions for $p^{ \pm}$when $s^{ \pm} \equiv s^{ \pm}(x)$ are not constants. Under steady state conditions the first equation implies that $j$ is a constant, and the boundary conditions imply that $j \equiv 0$. Therefore $s^{+} p^{+}=s^{-} p^{-}$, and the second equation reduces to

$$
\frac{\partial}{\partial x}\left(s^{+} p^{+}\right)=\left[\lambda \frac{s^{+}-s^{-}}{s^{+} s^{-}}\right] s^{+} p^{+} .
$$


This is a first-order equation for $s^{+} p^{+}$whose solution is

$$
p^{+}(x)=\frac{s^{+}(0) p^{+}(0)}{s^{+}(x)} \exp \left[\lambda \int_{0}^{x} \frac{s^{+}-s^{-}}{s^{+} s^{-}} \mathrm{d} \xi\right],
$$

and the condition of vanishing flux gives $p^{-}$. It follows that

$$
n(x)=c\left(\frac{1}{s^{+}(x)}+\frac{1}{s^{-}(x)}\right) \exp \left[\lambda \int_{0}^{x} \frac{s^{+}-s^{-}}{s^{+} s^{-}} \mathrm{d} \xi\right],
$$

where the constant $c$ is computed in such a way that $\int_{0}^{1} n(x) \mathrm{d} x$ is the total number of cells in the unit interval. From this one can determine how the distribution of $s^{ \pm}$ affects the distribution of $p$. In particular, if $s^{ \pm}$are not constant, then $p^{ \pm}$are also nonconstant. This is most easily seen if $s^{+}(x)=s^{-}(x)$, for then it follows directly from (3.13) that cells accumulate at the minima of the speed distribution. In any case, this simple model shows that cells can aggregate in a time-independent gradient by modifying only their speed.

One can also ask if cells can aggregate by maintaining a constant speed and modifying only their turning rate, in relation to either their spatial position, their direction of travel, or both. This will be analyzed in detail in the following section, but the essence of the effects can be understood as follows. Suppose that $s^{+}=s^{-}=s$ in (3.9) and that the turning rate depends on both position and direction of travel. Rewrite (3.9) in the form

$$
\begin{aligned}
& \frac{\partial p^{+}}{\partial t}+s \frac{\partial p^{+}}{\partial x}=-\lambda^{+} p^{+}+\lambda^{-} p^{-} \\
& \frac{\partial p^{-}}{\partial t}-s \frac{\partial p^{-}}{\partial x}=\lambda^{+} p^{+}-\lambda^{-} p^{-}
\end{aligned}
$$

where $\lambda^{ \pm}=\lambda^{ \pm}(x)$. Further, write

$$
\lambda^{ \pm}=\frac{\lambda^{+}+\lambda^{-}}{2} \pm \frac{\lambda^{+}-\lambda^{-}}{2} \equiv \lambda_{0} \pm \lambda_{1}
$$

and then (3.14) can be written in the equivalent form

$$
\begin{aligned}
\frac{\partial n}{\partial t}+\frac{\partial j}{\partial x} & =0, \\
\frac{\partial j}{\partial t}+2 \lambda_{0} j & =-s^{2} \frac{\partial n}{\partial x}-2 s \lambda_{1} n .
\end{aligned}
$$

Suppose that these equations are defined on the interval $[0,1]$ and that homogeneous Neumann data is imposed on the boundary. Under steady state conditions it follows directly that $j(x) \equiv 0$ and that

$$
n(x)=c \exp \left[-\frac{2}{s} \int_{0}^{x} \lambda_{1}(\xi) \mathrm{d} \xi\right],
$$

where the constant $c$ is computed in such a way that $\int_{0}^{1} n(x) \mathrm{d} x$ is the total number of cells in $[0,1]$. Consequently, there is no aggregation if $\lambda_{1} \equiv 0$. Therefore, if only the turning rate is spatially variable, then there must be a difference between the turning rates of left- and right-moving cells [47]. We can also formally define a 
chemotactic velocity in the limit in which the time derivative of $j$ is negligible, namely $u_{c}=-s \lambda_{1} / \lambda_{0}$, and if $\lambda_{1}$ is proportional to the gradient of an attractant or repellent field, the usual form given in (3.4) results.

The diffusion approximation to the transport equation in arbitrary space dimensions has been studied in [49], where it is shown that in general the bias in the turning rate must be an odd function of the velocity in order to obtain a nontrivial chemotactic velocity. Hillen and Stevens [33] have done a more complete analysis of hyperbolic chemotaxis equations in one space dimension and, in particular, prove local and global existence results for (3.9) when $s^{ \pm}$and $\lambda^{ \pm}$depend on the external signal and the system (3.9) is coupled with the parabolic equation for the external signal. As here, they conclude that two different mechanisms can lead to aggregation; namely,

- the turning rates of the right- and left-moving individuals are different;

- the speed $s$ depends on the external signal, which for time-independent nonconstant signals is equivalent to the statement that the speed of $s$ depends on the spatial variable $x$.

In reality, the speed of a bacterium is approximately constant, and, as we noted earlier, bacteria use modulation of the run length to aggregate. In order to understand how modulation of the turning behavior is related to the signal, we have to introduce the internal dynamics into the model. This is done in the next section.

3.3. Incorporation of internal dynamics. To have a predictive populationlevel model, one must first have a detailed model of a single cell of the type described earlier, and then one must lift this to the collective behavior of the population of individuals. To incorporate internal dynamics, we introduce the vector of internal state variables $y=\left(y_{1}, y_{2}, \ldots, y_{m}\right) \in \mathbb{R}^{m}$, which can include the concentration of receptors, proteins, etc. inside the cell, and we let $S(x, t)=\left(S_{1}, S_{2}, \ldots, S_{d}\right) \in \mathbb{R}^{d}$ denote the signals in the environment. The existing deterministic models (see section 2.1) of individual bacterial behavior can all be cast in the form of a system of ODEs that describe the evolution of the intracellular state, forced by the extracellular signal. Thus

$$
\frac{\mathrm{d} y}{\mathrm{~d} t}=f(y, S)
$$

where $f: \mathbb{R}^{m} \times \mathbb{R}^{d} \rightarrow \mathbb{R}^{m}$ describes the particular model. We showed in [21] that for noninteracting walkers such as bacteria, the internal dynamics can be incorporated in the transport equation as follows. Let $p(x, v, y, t)$ be the density of bacteria in a $(2 N+m)$-dimensional phase space with coordinates $(x, v, y)$, where $x \in \mathbb{R}^{N}$ is the position of a cell, $v \in V \subset \mathbb{R}^{N}$ is its velocity, and $y \in Y \subset \mathbb{R}^{m}$ is its internal state, which evolves according to (3.16). The evolution of $p$ is governed by the transport equation

$$
\frac{\partial p}{\partial t}+\nabla_{x} \cdot v p+\nabla_{y} \cdot f p=-\lambda(y) p+\int_{V} \lambda(y) T\left(v, v^{\prime}, y\right) p\left(x, v^{\prime}, y, t\right) \mathrm{d} v^{\prime}
$$

(see [21, section 3]). Here, as before, we assume that the random velocity changes are the result of a Poisson process of intensity $\lambda(y)$. The kernel $T\left(v, v^{\prime}, y\right)$ gives the probability of a change in velocity from $v^{\prime}$ to $v$, given that a reorientation occurs. The kernel $T$ is nonnegative and satisfies the normalization condition $\int_{V} T\left(v, v^{\prime}, y\right) \mathrm{d} v=1$. The objective is to derive an evolution equation for the macroscopic density

$$
n(x, t)=\int_{Y} \int_{V} p(x, v, y, t) \mathrm{d} v \mathrm{~d} y
$$


of individuals. As we discussed earlier, two essential components in the chemotactic response to an attractant are the rapid decrease in the motor control protein $\mathrm{CheY}_{\mathrm{p}}$ and the slow return of this protein to its prestimulus level in the face of a constant stimulus (cf. Figure 2.2). Therefore, as a first step toward understanding how internal dynamics can be incorporated into macroscopic descriptions, we consider a highly simplified model of signal transduction which has similar properties to the realistic signal transduction models described earlier.

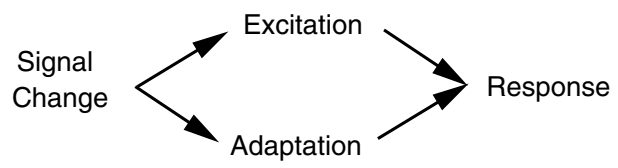

FIG. 3.1. A schematic of the essential components of signal transduction in an adapting system. Usually, excitation is much faster than adaptation.

3.3.1. Cartoon internal dynamics and their properties. Suppose that the temporal variation of the chemokinetic signal seen by a bacterium is given by $C(t)$. The major components of signal transduction that must be incorporated in the cartoon model are the excitation and adaptation steps, as illustrated schematically in Figure 3.1. This schematic can be translated into a linear model as follows [47]. Suppose that there are two internal state variables $y_{1}$ and $y_{2}$ and that these variables evolve according to the system of equations

$$
\frac{\mathrm{d} y_{1}}{\mathrm{~d} t}=\frac{C(t)-\left(y_{1}+y_{2}\right)}{t_{e}}, \quad \frac{\mathrm{d} y_{2}}{\mathrm{~d} t}=\frac{C(t)-y_{2}}{t_{a}} .
$$

The magnitude of $t_{e}$ reflects the time scale for excitation, and the magnitude of $t_{a}$ reflects the time scale for adaptation.

If the signal is transmitted via receptors, and if we assume that binding equilibrates rapidly and the signal is a given function of the fraction of receptors bound, then the input signal $C(t)$ will have the form

$$
C(t)=G\left(\frac{S(x, t)}{K_{D}+S(x, t)}\right) \equiv g(S(x, t)),
$$

where $G(\cdot)$ is a given function, $S(x, t)$ is the extracellular signal at time $t$ and the current position of cell $x \equiv x(t)$, and $K_{D}$ is the binding constant [8].

Next, we suppose that the deviation from the basal response is a function of $y_{1}$. Then this simple scheme can be viewed as having two input pathways, an excitatory one that stimulates the production of $y_{1}$, and hence increases the response, and an inhibitory one that increases the production of $y_{2}$, which in turn shuts off the response. We call $y_{1}$ the excitation or controller variable, because later it will be used to control the turning rate.

Since this system is linear the solution can be obtained analytically, and for the special case in which $y_{1}(0)=y_{2}(0)=0$ and $C(t)$ is a step function of amplitude from 0 to $C_{1}$ that turns on at $t=0$, the solution is as follows:

$$
y_{1}=\frac{C_{1} t_{a}}{t_{a}-t_{e}}\left(e^{-t / t_{a}}-e^{-t / t_{e}}\right), \quad y_{2}=C_{1}\left(1-e^{-t / t_{a}}\right) .
$$

From this one sees that if $t_{e} \ll t_{a}$, then for $t \gg t_{e}, y_{1}$ relaxes to

$$
y_{1}(t) \sim C_{1} e^{-t / t_{a}} \equiv C_{1}-y_{2}(t) .
$$


This is just the pseudo-steady-state value of $y_{1}$ which one gets by setting $d y_{1} / d t=0$. We note from (3.19) that when the stimulus $C(t)$ is constant the steady state level of $y_{1}$ is zero, i.e., the response adapts perfectly to any constant stimulus, but the level of $y_{2}$ does not adapt.

The typical response for a single step in the stimulus from 0 to $C_{1}$ when $t_{e}<t_{a}$ is shown in Figure 3.2(a), where one can see that neither $y_{1}$ nor $y_{2}$ exceeds $C_{1}$. The response to two-step changes that are well separated compared to the adaptation time are shown Figure 3.2(b).

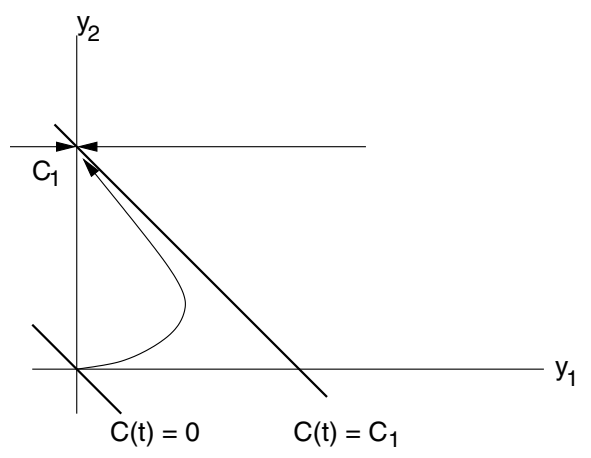

(a)

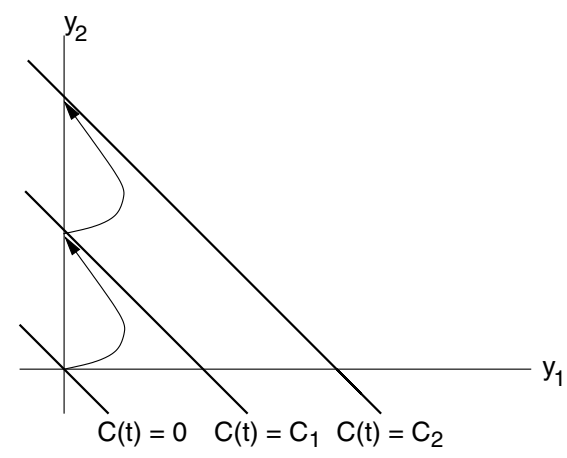

(b)

FIG. 3.2. The phase plane for the model adapting system described by (3.19).

To understand the role played by adaptation on the level of the controller variable, we consider the time course of $y_{1}$ in a cell moving in a static gradient. If excitation is rapid compared to adaptation and the change in the signal, we may set $y_{1}=$ $C(t)-y_{2}=t_{a} \frac{d y_{2}}{d t}$ and thus have to solve only for $y_{2}$ along a specified path. Thus it is convenient to differentiate the second equation in (3.19) and to solve the resulting equation for $\frac{d y_{2}}{d t}$. We find that $u \equiv \frac{d y_{2}}{d t}$ satisfies

$$
\frac{\mathrm{d} u}{\mathrm{~d} t}+\frac{1}{t_{a}} u=\frac{1}{t_{a}} \frac{\mathrm{d} C}{\mathrm{~d} t} .
$$

For a cell that begins at $x_{0}$ and moves with a velocity $v$ through a time-invariant concentration field $S(x)$, we have

$$
\frac{\mathrm{d} C}{\mathrm{~d} t}=g^{\prime}\left(S\left(x_{0}+v t\right)\right) v \cdot \nabla S,
$$

and using this (3.22) can be solved for any specified cell path. Note that if $t_{a}=\infty$, then $y_{2}$ is identically constant and $y_{1}$ simply tracks the stimulus. To show that adaptation can play a significant role in the motion of a cell, we suppose that $S(x)$ varies linearly and that $g$ is the identity, and we consider an infinite domain. Since the signal distribution is linear and $g^{\prime}=1$, the gradient $S^{\prime}$ is constant, and the right-hand side of (3.22) is constant.

Suppose that cells move at a constant speed for a fixed time $\tau$, at which point they choose a new direction randomly. One finds that

$$
u(\tau)=e^{-\tau / t_{a}} u(0) \pm \omega h(\tau),
$$

where

$$
\omega \equiv|v| S^{\prime} \quad \text { and } \quad h(\tau)=\left(1-e^{-\tau / t_{a}}\right) .
$$


Therefore, at the end of the first step the initial level is decremented by the exponential factor $\gamma_{0}=e^{-\tau / t_{a}}$ and an amount $\gamma_{1}=\omega h(\tau)$ is added or subtracted, depending on the direction of motion. If the step length is constant, the controller level after $n$ steps is the solution of the difference equation

$$
u_{n} \equiv u(n \tau)=\gamma_{0} u_{n-1} \pm \gamma_{1},
$$

and it follows that the solution is given by

$$
u_{n}=\gamma_{0}^{n} u_{0}+\gamma_{1}\left[ \pm \gamma_{0}^{n-1} \pm \gamma_{0}^{n-2}+\cdots \pm \gamma_{0}\right] .
$$

If the choice of direction is unbiased, the expected value of the quantity in brackets is zero, since the walk is then symmetric. However, if the run time, and hence the step length, is not fixed at $\tau$ but is biased appropriately, the outcome is different.

To see this consider two, two-step realizations of this process. Suppose that the first cell first moves to the right and then to the left, whereas the second cell first moves to the left and then to the right. The controller level in the first cell at time $2 \tau$ is

$$
\begin{aligned}
u^{-}(2 \tau) & =e^{-\tau / t_{a}} u^{+}(\tau)-\omega h(\tau) \\
& =e^{-2 \tau / t_{a}} u(0)-\omega h^{2}(\tau),
\end{aligned}
$$

whereas the level in the second cell is

$$
\begin{aligned}
u^{+}(2 \tau) & =e^{-\tau / t_{a}} u^{-}(\tau)+\omega h(\tau) \\
& =e^{-2 \tau / t_{a}} u(0)+\omega h^{2}(\tau) .
\end{aligned}
$$

Here the superscript denotes that direction of travel at the current time or the time just prior to a turn. From these results one sees that the outcome is not symmetric, and, in fact, the cell that most recently moves up-gradient has the higher level of controller. In other words, the cells carry memory of their paths with them, and the most recent portion of the path is most important. If, for instance, the turning rate depends on the controller, then the turning rate becomes a function of the past history of the cell, as is needed in the bacterial example. In particular, if the turning rate as defined later is of the form $\lambda=\lambda_{0}-b y_{1}$, then the cell moving leftward at $2 \tau$ has a higher probability of turning than does the cell moving rightward at $2 \tau$. From this observation (together with the conclusions of section 3.2.1), one can formulate an heuristic argument that shows that adaptation may be important in determining whether or not cells aggregate at the high point of an attractant.

In contrast, when adaptation is infinitely slow, then we may take $y_{2}=0$ and $y_{1}=C(t)$. In this case the controller variable tracks the external signal with perfect fidelity, and, as a result, there is no dependence of the controller level on the path. As we showed in section 3.2.1, when the controller affects only the turning rate, this cannot lead to aggregation. For any finite rate of adaptation the difference between the controller level in the rightward and leftward moving cells at $2 \tau$ is $2 \omega h^{2}(\tau)$, which goes to zero as $t_{a} \rightarrow \infty$.

This discussion, which was based on individual paths of bacteria, suggests that excitation and adaptation are essential components for aggregation of bacterial populations. To make the connection between microscopic models and the classical macroscopic chemotaxis equations rigorous, we briefly describe the approach developed in $[21]$. 
3.4. Asymptotic methods for deriving the chemotaxis equation with internal dynamics. A macroscopic evolution equation for the cell density $n$ in one dimension that incorporates taxis based on internal dynamics and no interaction between walkers was first derived in [21]. Let $p^{ \pm}(x, y, t)$ be the density of walkers that are at $(x, t)$ with the internal state $y$ and are moving to the right (left), and suppose that the internal state evolves according to (3.16). Then $p^{ \pm}(x, y, t)$ satisfy the equations

$$
\begin{gathered}
\frac{\partial p^{+}}{\partial t}+s \frac{\partial p^{+}}{\partial x}+\sum_{i=1}^{m} \frac{\partial}{\partial y_{i}}\left[f_{i}(y, S) p^{+}\right]=\lambda(y)\left[-p^{+}+p^{-}\right] \\
\frac{\partial p^{-}}{\partial t}-s \frac{\partial p^{-}}{\partial x}+\sum_{i=1}^{m} \frac{\partial}{\partial y_{i}}\left[f_{i}(y, S) p^{-}\right]=\lambda(y)\left[p^{+}-p^{-}\right] .
\end{gathered}
$$

In [21] we used the cartoon model (3.19)-(3.20) of internal dynamics, and we supposed that the signal $S$ was time-independent. Thus the governing equations for the internal state are (3.19) with $C(t)$ replaced by $g(S(x))$. We identified the response with the turning frequency $\lambda(y)$ and assumed linear dependence of $\lambda$ on $y_{1}$ :

$$
\lambda(y) \equiv \text { Response }=\lambda_{0}-b y_{1},
$$

where $\lambda_{0}$ is the basal turning frequency for a fully adapted cell and $b$ is a positive constant. The term $b y_{1}$ describes the change in the turning frequency in response to a signal, and the negative sign accounts for the fact that an increase of $y_{1}$ should produce a decrease in turning rate. The linear turning rate (3.32) is reasonable for shallow gradients of the signal, which is the case treated in detail in [21]. Under these assumptions, we obtained the hyperbolic chemotaxis equation

$$
\frac{\partial^{2} n}{\partial t^{2}}+2 \lambda_{0} \frac{\partial n}{\partial t}=\frac{\partial}{\partial x}\left(s^{2} \frac{\partial n}{\partial x}-g^{\prime}(S(x)) \frac{2 b s^{2} t_{a}}{\left(1+2 \lambda_{0} t_{a}\right)\left(1+2 \lambda_{0} t_{e}\right)} S^{\prime}(x) n\right)
$$

using a hyperbolic scaling of time and space and obtained the classical chemotaxis equation (3.5), valid for large time,

$$
\frac{\partial n}{\partial t}=\frac{\partial}{\partial x}\left(\frac{s^{2}}{2 \lambda_{0}} \frac{\partial n}{\partial x}-g^{\prime}(S(x)) \frac{b s^{2} t_{a}}{\lambda_{0}\left(1+2 \lambda_{0} t_{a}\right)\left(1+2 \lambda_{0} t_{e}\right)} S^{\prime}(x) n\right)
$$

using a parabolic scaling of space and time (these scalings are defined later). This equation was derived for shallow gradients of the signal and may not even apply for large gradients, but that form is widely used for macroscopic models. In either the form (3.33) or (3.34) one can identify the chemotactic sensitivity (3.4) as

$$
\chi(S)=g^{\prime}(S(x)) \frac{b s^{2} t_{a}}{\lambda_{0}\left(1+2 \lambda_{0} t_{a}\right)\left(1+2 \lambda_{0} t_{e}\right)},
$$

and this clearly depends on parameters of the internal dynamics.

Next, we address the derivation of chemotaxis equations in two and three space dimensions, which is not a straightforward extension of the derivation in one dimension because additional velocity moments appear. We show here two methods to treat this problem leading to multidimensional counterparts of (3.33) and (3.34). The first method, based on the ideas from [21] and [32, 49], leads to the parabolic classical chemotaxis equation. In section 4 , we derive a macroscopic equation using a very general 
setup and derive the classical chemotaxis equation (4.44) for bacterial movement from the general theory. In section 5, we discuss another approach of deriving the macroscopic equations based on ideas from [21] and [31]. This leads to a multidimensional form of (3.33).

4. Chemotaxis equations from the transport equation in higher dimensions. In order to derive a macroscopic equation for the density $n$ (given by (3.18)) from the transport equation (3.17), we have to specify the internal dynamics $f(y, S)$, the turning rate $\lambda(y)$, and the turning kernel $T\left(v, v^{\prime}, y\right)$. As before, we use the cartoon internal dynamics (3.19)-(3.20), the linear turning rate (3.32), and a space-dependent but time-independent scalar signal $S$. We introduce new internal variables $\left(z_{1}, z_{2}\right)$ that measure deviations from the steady state, and hence vary over $R^{2}$, by

$$
y_{1}=z_{1}, \quad y_{2}=g(S(x))+z_{2} \quad \Leftrightarrow \quad z_{1}=y_{1}, \quad z_{2}=y_{2}-g(S(x)) .
$$

Then the internal dynamics (3.19)-(3.20) and the turning rate (3.32) are now

$\frac{\mathrm{d} z_{2}}{\mathrm{~d} t}=\frac{\mathrm{d} y_{2}}{\mathrm{~d} t}-\frac{\mathrm{d} g(S(x))}{\mathrm{d} t}=\frac{g(S(x))-y_{2}}{t_{a}}-g^{\prime}(S(x)) \nabla_{x} S \cdot v=-\frac{z_{2}}{t_{a}}-g^{\prime}(S(x)) \nabla_{x} S \cdot v$,

$$
\lambda(y) \equiv \lambda(z)=\lambda_{0}-b z_{1},
$$

where $v$ is the velocity of the cell, $S: \mathbb{R}^{N} \rightarrow[0, \infty)$ is the external signal, and $t_{a}, t_{e}$, $\lambda_{0}$, and $b$ are constants satisfying $t_{a}>t_{e} \geq 0, \lambda_{0}>0$, and $b>0$.

In general the turning kernel might depend on the internal state, for example if cells are permanently polarized, but here we assume that it is independent of the internal state, which is the case for bacterial chemotaxis. In view of the previous simplifications, the transport equation (3.17) now reads as

$$
\begin{gathered}
\frac{\partial p}{\partial t}+\nabla_{x} \cdot v p+\frac{\partial}{\partial z_{1}}\left[\left(\frac{-z_{1}-z_{2}}{t_{e}}\right) p\right]+\frac{\partial}{\partial z_{2}}\left[\left(-\frac{z_{2}}{t_{a}}-g^{\prime}(S(x)) \nabla_{x} S \cdot v\right) p\right] \\
=-\left(\lambda_{0}-b z_{1}\right) p+\int_{V}\left(\lambda_{0}-b z_{1}\right) T\left(v, v^{\prime}\right) p\left(x, v^{\prime}, z, t\right) \mathrm{d} v^{\prime}
\end{gathered}
$$

where $p \equiv p(x, v, z, t)$ is the number density of individuals at point $x$ with velocity $v$ and with internal state $z=\left(z_{1}, z_{2}\right)$ at time $t$. To simplify the notation, we will drop the index $x$ in $\nabla_{x}$, and from now on we will denote the spatial gradient by $\nabla \equiv \nabla_{x}$. Later we stipulate precise restrictions on the kernel.

To find the macroscopic evolution equation(s) for the density $n(x, t)$, we define the following moments with respect to internal variables: ${ }^{1}$

$$
\begin{gathered}
M(x, v, t)=\int_{\mathbb{R}^{2}} p(x, v, z, t) \mathrm{d} z, \\
M_{1}(x, v, t)=\int_{\mathbb{R}^{2}} z_{1} p(x, v, z, t) \mathrm{d} z,
\end{gathered}
$$

\footnotetext{
${ }^{1}$ We assume throughout that the initial data has compact support in $(x, v, z)$-space, and therefore the following moments are well defined.
} 


$$
M_{2}(x, v, t)=\int_{\mathbb{R}^{2}} z_{2} p(x, v, z, t) \mathrm{d} z
$$

$$
M_{11}(x, v, t)=\int_{\mathbb{R}^{2}}\left(z_{1}\right)^{2} p(x, v, z, t) \mathrm{d} z \quad \text { and } \quad M_{12}(x, v, t)=\int_{\mathbb{R}^{2}} z_{1} z_{2} p(x, v, z, t) \mathrm{d} z .
$$

Then multiplying (4.5) by $1, z_{1}$, and $z_{2}$, respectively, and integrating the resulting equations with respect to $z=\left(z_{1}, z_{2}\right)$, we obtain

$$
\begin{gathered}
\frac{\partial M}{\partial t}+\nabla \cdot v M=-\lambda_{0} M+\lambda_{0} \int_{V} T\left(v, v^{\prime}\right) M\left(x, v^{\prime}, t\right) \mathrm{d} v^{\prime} \\
+b M_{1}-b \int_{V} T\left(v, v^{\prime}\right) M_{1}\left(x, v^{\prime}, t\right) \mathrm{d} v^{\prime} \\
\frac{\partial M_{1}}{\partial t}+\nabla \cdot v M_{1}=-\left(\lambda_{0}+\frac{1}{t_{e}}\right) M_{1}-\frac{1}{t_{e}} M_{2}+\lambda_{0} \int_{V} T\left(v, v^{\prime}\right) M_{1}\left(x, v^{\prime}, t\right) \mathrm{d} v^{\prime} \\
+b M_{11}-b \int_{V} T\left(v, v^{\prime}\right) M_{11}\left(x, v^{\prime}, t\right) \mathrm{d} v^{\prime}
\end{gathered}
$$

$$
\begin{aligned}
\frac{\partial M_{2}}{\partial t}+\nabla \cdot v M_{2}= & -g^{\prime}(S(x)) \nabla S \cdot v M-\left(\lambda_{0}+\frac{1}{t_{a}}\right) M_{2}+\lambda_{0} \int_{V} T\left(v, v^{\prime}\right) M_{2}\left(x, v^{\prime}, t\right) \mathrm{d} v^{\prime} \\
& +b M_{12}-b \int_{V} T\left(v, v^{\prime}\right) M_{12}\left(x, v^{\prime}, t\right) \mathrm{d} v^{\prime} .
\end{aligned}
$$

Next, we have two possibilities: either we can integrate the equations (4.10)-(4.12) with respect to $v$ to derive moment equations, as was done for one dimension in [21], or we can use a scaling argument and asymptotic analysis. The moment approach introduces higher-order velocity moments into the moment equations, and, consequently, we cannot use the one-dimensional method directly from [21]. We will discuss this issue in section 5: here, we begin with the parabolic scaling argument. First of all, we restrict consideration to the case of shallow gradients of the signal. Roughly speaking, if the gradient of the signal is small, then the new internal variables $\left(z_{1}, z_{2}\right)$ are close to zero, and, consequently, the higher-order moments $M_{11}, M_{12}$ are much smaller than the lower-order moments and can be neglected (see [21, Lemma 6.2]). Hence (4.10)-(4.12) lead to the following equations:

$$
\begin{gathered}
\frac{\partial M}{\partial t}+\nabla \cdot v M=-\lambda_{0} M+\lambda_{0} \int_{V} T\left(v, v^{\prime}\right) M\left(x, v^{\prime}, t\right) \mathrm{d} v^{\prime} \\
+b M_{1}-b \int_{V} T\left(v, v^{\prime}\right) M_{1}\left(x, v^{\prime}, t\right) \mathrm{d} v^{\prime} \\
\frac{\partial M_{1}}{\partial t}+\nabla \cdot v M_{1}=-\left(\lambda_{0}+\frac{1}{t_{e}}\right) M_{1}-\frac{1}{t_{e}} M_{2}+\lambda_{0} \int_{V} T\left(v, v^{\prime}\right) M_{1}\left(x, v^{\prime}, t\right) \mathrm{d} v^{\prime}
\end{gathered}
$$

$\frac{\partial M_{2}}{\partial t}+\nabla \cdot v M_{2}=-g^{\prime}(S(x)) \nabla S \cdot v M-\left(\lambda_{0}+\frac{1}{t_{a}}\right) M_{2}+\lambda_{0} \int_{V} T\left(v, v^{\prime}\right) M_{2}\left(x, v^{\prime}, t\right) \mathrm{d} v^{\prime}$.

Now we consider a general turning kernel with the technical properties given in section 4.1 and derive a general macroscopic equation for the cellular density $n(x, t)$ based on these. 
4.1. Properties of the turning kernel. The standing assumptions on the turning kernel $T\left(v, v^{\prime}\right)$ and their mathematical consequences are stated here without proof. The interested reader can consult [32] for the proofs of results in this section. Suppose that $v \in V$, where $V \subset \mathbb{R}^{N}$ is a bounded set symmetric with respect to the origin. Denote $\mathbb{K}=\left\{u \in L^{2}(V) \mid u \geq 0\right\}$, and let $T \in L^{2}(V \times V)$ be such that

$$
T\left(v, v^{\prime}\right) \geq 0, \quad \int_{V} T\left(v, v^{\prime}\right) \mathrm{d} v=1 .
$$

The assumptions (4.16) are natural, as $T$ denotes a probability of the velocity jump from $v^{\prime}$ to $v$, given that the jump occurs. We will make use of the following assumptions on the kernel $T$ :

(A1) There are functions $u_{0} \not \equiv 0, \phi$, and $\psi \in \mathbb{K}$ with the properties that $\phi$ vanishes at most on a set of Lebesgue measure zero and such that for all $\left(v, v^{\prime}\right) \in V \times V$ we have $u_{0}(v) \phi\left(v^{\prime}\right) \leq T\left(v^{\prime}, v\right) \leq u_{0}(v) \psi\left(v^{\prime}\right)$.

(A2) Denote $\mathcal{Z}=\left\{f \in L^{2}(V) \mid \int_{V} f(v) \mathrm{d} v=0\right\}$. Then

$$
\sup _{f \in \mathcal{Z}, f \neq 0} \frac{\int_{V}\left(T\left(v, v^{\prime}\right) f\left(v^{\prime}\right)\right)^{2} \mathrm{~d} v^{\prime}}{\int_{V} f^{2}(v) \mathrm{d} v}<1 .
$$

(A3) $\int_{V} T\left(v, v^{\prime}\right) \mathrm{d} v^{\prime}=1$.

Define the integral operators $\mathcal{T}: \mathbb{K} \rightarrow \mathbb{K}$ and $\mathcal{T}^{*}: \mathbb{K} \rightarrow \mathbb{K}$ as

$$
\mathcal{T} f=\int_{V} T\left(v, v^{\prime}\right) f\left(v^{\prime}\right) \mathrm{d} v^{\prime}, \quad \mathcal{T}^{*} f=\int_{V} T\left(v^{\prime}, v\right) f\left(v^{\prime}\right) \mathrm{d} v^{\prime} .
$$

Note that $\mathcal{T}$ and $\mathcal{T}^{*}$ are compact and that their spectral radius is 1 . By virtue of (4.16) and (A3), both $\mathcal{T}$ and $\mathcal{T}^{*}$ have an eigenvalue 1 . For our purposes, it will be convenient to define the operator $\mathcal{A}: L^{2}(V) \rightarrow L^{2}(V)$ and its adjoint $\mathcal{A}^{*}: L^{2}(V) \rightarrow L^{2}(V)$ as follows:

$$
\mathcal{A} f=-f+\mathcal{T} f, \quad \mathcal{A}^{*} f=-f+\mathcal{T}^{*} f .
$$

The properties of $\mathcal{A}$ and $\mathcal{A}^{*}$ are given in the following theorem.

TheOREM 4.1. Let us suppose that the turning kernel $T \in L^{2}(V \times V)$ satisfies (4.16) and (A1)-(A3). Then we have the following:

(i) 0 is a simple eigenvalue of $\mathcal{A}$ and the corresponding eigenfunction is $\phi(v) \equiv 1$.

(ii) Let us denote $\mu_{2}=1-\|\mathcal{T}\|_{\langle 1\rangle}{ }^{\perp}$. Then all nonzero eigenvalues satisfy $-2<$ Re $\mu<-\mu_{2}<0$, and to within a scalar factor there is no other positive eigenfunction.

(iii) There is a decomposition $L^{2}(V)=\langle 1\rangle \oplus\langle 1\rangle^{\perp}$, and we have the estimate

$$
\int_{V} \psi \mathcal{A} \psi d v \leq-\mu_{2}\|\psi\|_{L^{2}(V)}^{2} \quad \text { for all } \psi \in\langle 1\rangle^{\perp} .
$$

Proof. See [32, Theorem 2.4].

4.2. Parabolic limit. To study the macroscopic behavior for large times one can use the parabolic scaling of space and time, as described in [32, 49] and [21]. Let $T=1 \mathrm{sec}, L=1 \mathrm{~mm}$, and $s_{0}=10 \mu / \mathrm{sec}$ be the characteristic scales for space, time, and velocity, respectively. Let $N_{0}$ be a scale factor for the particle density, and define the dimensionless variables

$\hat{x}=\frac{x}{L}, \quad \hat{t}=\frac{t}{T}, \quad \hat{v}=\frac{v}{s_{0}}, \quad \hat{M}=\frac{M}{N_{0}}, \quad \hat{M}_{1}=\frac{M_{1}}{N_{0}}, \quad$ and $\quad \hat{M}_{2}=\frac{M_{2}}{N_{0}}$. 
Denote $\hat{\lambda}_{0} \equiv \lambda_{0} T, \hat{b} \equiv b T$, and $\hat{t}_{a} \equiv \frac{t_{a}}{T}$ and introduce the scaling parameter $\varepsilon$. The parabolic scales of space and time are given by

$$
T_{1}=\frac{1}{\varepsilon^{2}} T, \quad L_{1}=\frac{1}{\varepsilon} \frac{s_{0}}{L / T} L,
$$

and using these the dimensionless equations take the form

$$
\begin{aligned}
\varepsilon^{2} \frac{\partial \hat{M}}{\partial \hat{t}}+\varepsilon \nabla \cdot \hat{v} \hat{M}= & -\hat{\lambda}_{0} \hat{M}+\hat{b} \hat{M}_{1}+\hat{\lambda}_{0} \int_{V} \hat{T}\left(\hat{v}, \hat{v}^{\prime}\right) \hat{M}\left(\hat{x}, \hat{v}^{\prime}, \hat{t}\right) d \hat{v}^{\prime} \\
& -\hat{b} \int_{V} \hat{T}\left(\hat{v}, \hat{v}^{\prime}\right) \hat{M}_{1}\left(\hat{x}, \hat{v}^{\prime}, \hat{t}\right) d \hat{v}^{\prime}
\end{aligned}
$$

$$
\begin{aligned}
\varepsilon^{2} \frac{\partial \hat{M}_{1}}{\partial \hat{t}}+\varepsilon \nabla \cdot \hat{v} \hat{M}_{1}=-\left(\hat{\lambda}_{0}+\right. & \left.\frac{1}{\hat{t}_{e}}\right) \hat{M}_{1}-\frac{1}{\hat{t}_{e}} \hat{M}_{2}+\hat{\lambda}_{0} \int_{V} \hat{T}\left(\hat{v}, \hat{v}^{\prime}\right) \hat{M}_{1}\left(\hat{x}, \hat{v}^{\prime}, \hat{t}\right) d \hat{v}^{\prime}, \\
\varepsilon^{2} \frac{\partial \hat{M}_{2}}{\partial \hat{t}}+\varepsilon \nabla \cdot \hat{v} \hat{M}_{2}= & -\varepsilon g^{\prime}(\hat{S}(\hat{x})) \nabla \hat{S} \cdot \hat{v} \hat{M}-\left(\hat{\lambda}_{0}+\frac{1}{\hat{t}_{a}}\right) \hat{M}_{2} \\
& +\hat{\lambda}_{0} \int_{V} \hat{T}\left(\hat{v}, \hat{v}^{\prime}\right) \hat{M}_{2}\left(\hat{x}, \hat{v}^{\prime}, \hat{t}\right) d \hat{v}^{\prime} .
\end{aligned}
$$

For simplicity, we drop the hats in (4.20)-(4.22) and use the notation (4.17) to rewrite (4.20)-(4.22) in the following form:

$$
\begin{gathered}
\varepsilon^{2} \frac{\partial M}{\partial t}+\varepsilon \nabla \cdot v M=\lambda_{0} \mathcal{A} M-b \mathcal{A} M_{1}, \\
\varepsilon^{2} \frac{\partial M_{1}}{\partial t}+\varepsilon \nabla \cdot v M_{1}=\lambda_{0} \mathcal{A} M_{1}-\frac{1}{t_{e}} M_{1}-\frac{1}{t_{e}} M_{2}, \\
\varepsilon^{2} \frac{\partial M_{2}}{\partial t}+\varepsilon \nabla \cdot v M_{2}=-\varepsilon g^{\prime}(S(x)) \nabla S \cdot v M+\lambda_{0} \mathcal{A} M_{2}-\frac{1}{t_{a}} M_{2} .
\end{gathered}
$$

As was argued in [21], one can assume that all dimensionless parameters in (4.23)(4.25) are $\mathcal{O}(1)$. Moreover, assuming the regular perturbation expansion

$$
\begin{aligned}
& M=M^{0}+\varepsilon M^{1}+\varepsilon^{2} M^{2}+\cdots, \quad M_{1}=M_{1}^{0}+\varepsilon M_{1}^{1}+\varepsilon^{2} M_{1}^{2}+\cdots, \\
& M=M_{2}^{0}+\varepsilon M_{2}^{1}+\varepsilon^{2} M_{2}^{2}+\cdots,
\end{aligned}
$$

substituting this into (4.23)-(4.25), and comparing terms of equal order in $\varepsilon$, we obtain

$$
\begin{aligned}
\varepsilon^{0}: \quad & \lambda_{0} \mathcal{A} M^{0}-b \mathcal{A} M_{1}^{0}=0, \\
& \lambda_{0} \mathcal{A} M_{1}^{0}-\frac{1}{t_{e}} M_{1}^{0}-\frac{1}{t_{e}} M_{2}^{0}=0, \\
& \lambda_{0} \mathcal{A} M_{2}^{0}-\frac{1}{t_{a}} M_{2}^{0}=0, \\
\varepsilon^{1}: \quad & \nabla \cdot v M^{0}=\lambda_{0} \mathcal{A} M^{1}-b \mathcal{A} M_{1}^{1}, \\
& \nabla \cdot v M_{1}^{0}=\lambda_{0} \mathcal{A} M_{1}^{1}-\frac{1}{t_{e}} M_{1}^{1}-\frac{1}{t_{e}} M_{2}^{1}, \\
& \nabla \cdot v M_{2}^{0}+g^{\prime}(S(x)) \nabla S \cdot v M^{0}=\lambda_{0} \mathcal{A} M_{2}^{1}-\frac{1}{t_{a}} M_{2}^{1}, \\
& \frac{\partial M^{0}}{\partial t}+\nabla_{x} \cdot v M^{1}=\mathcal{A}\left(\lambda_{0} M^{2}-b M_{1}^{2}\right) .
\end{aligned}
$$


By virtue of Theorem 4.1, the operator $\mathcal{A}$ has no positive eigenvalues, and, consequently, (4.28) implies that $M_{2}^{0}=0$. Similarly, (4.27) implies that $M_{1}^{0}=0$. Finally, using Theorem 4.1 again, we see that $M^{0}$ is independent of velocity, i.e., $M^{0} \equiv M^{0}(x, t)$. Therefore (4.30)-(4.31) give

$$
M_{1}^{1}=\left(t_{e} \lambda_{0} \mathcal{A}-I\right)^{-1} M_{2}^{1} \quad \text { and } \quad M_{2}^{1}=t_{a}\left(t_{a} \lambda_{0} \mathcal{A}-I\right)^{-1} g^{\prime}(S(x)) \nabla S \cdot v M^{0} .
$$

From (4.29) we obtain

$$
\mathcal{A} M^{1}=\frac{1}{\lambda_{0}} \nabla \cdot v M^{0}+\frac{b t_{a}}{\lambda_{0}} \mathcal{A}\left(t_{e} \lambda_{0} \mathcal{A}-I\right)^{-1}\left(t_{a} \lambda_{0} \mathcal{A}-I\right)^{-1} g^{\prime}(S(x)) \nabla S \cdot v M^{0} .
$$

Theorem 4.1 guarantees that the inverses of the operators $\left(t_{e} \lambda_{0} \mathcal{A}-I\right)$ and $\left(t_{a} \lambda_{0} \mathcal{A}-I\right)$ exist, but $\mathcal{A}$ is singular. However, as 0 is a simple eigenvalue of $\mathcal{A}$, one can define the pseudoinverse operator $\mathcal{B}$ as

$$
\mathcal{B}=\left(\left.\mathcal{A}\right|_{\langle 1\rangle^{\perp}}\right)^{-1}
$$

and then (4.33) reads as

$$
M^{1}=\mathcal{B} \frac{1}{\lambda_{0}} \nabla \cdot v M^{0}+\frac{b t_{a}}{\lambda_{0}}\left(t_{e} \lambda_{0} \mathcal{A}-I\right)^{-1}\left(t_{a} \lambda_{0} \mathcal{A}-I\right)^{-1} g^{\prime}(S(x)) \nabla S \cdot v M^{0}+C,
$$

where $C \in\langle 1\rangle$; i.e., $C$ is independent of $v$. Finally, (4.32) implies that the left-hand side

$$
\frac{\partial M^{0}}{\partial t}+\nabla \cdot v M^{1}
$$

is in the range of the operator $\mathcal{A}$. Consequently, using a Fredholm alternative, the left-hand side must be $L^{2}$-orthogonal to the constant function, i.e.,

$$
\int_{V} \frac{\partial M^{0}}{\partial t}+\nabla \cdot v M^{1} \mathrm{~d} v=0
$$

This, together with (4.34), implies that

$$
\begin{aligned}
|V| \frac{\partial M^{0}}{\partial t} & +\frac{1}{\lambda_{0}} \nabla \cdot \int_{V} v \mathcal{B} v \cdot \nabla M^{0} \mathrm{~d} v \\
& +\frac{b t_{a}}{\lambda_{0}} \nabla \cdot \int_{V} v\left(t_{e} \lambda_{0} \mathcal{A}-I\right)^{-1}\left(t_{a} \lambda_{0} \mathcal{A}-I\right)^{-1} v \cdot g^{\prime}(S(x)) M^{0} \nabla S \mathrm{~d} v=0 .
\end{aligned}
$$

Note that the term $\int_{V} \nabla \cdot v C \mathrm{~d} v$ vanishes, as $C$ is independent of $v$. If we define the diffusion tensor and the chemotactic tensor as

$$
\begin{gathered}
D=-\frac{1}{|V| \lambda_{0}} \int_{V} v \otimes \mathcal{B} v \mathrm{~d} v \\
\chi(S)=\frac{b t_{a}}{|V| \lambda_{0}} g^{\prime}(S(x)) \int_{V} v \otimes\left(t_{e} \lambda_{0} \mathcal{A}-I\right)^{-1}\left(t_{a} \lambda_{0} \mathcal{A}-I\right)^{-1} v \mathrm{~d} v,
\end{gathered}
$$


then (4.35) reads as

$$
\frac{\partial M^{0}}{\partial t}=\nabla \cdot\left(D \nabla M^{0}-M^{0} \chi(S) \nabla S\right)
$$

Finally, (3.18) implies that the density of cells $n$ is given by

$$
n(x, t)=\int_{Z} \int_{V} p(x, v, z, t) \mathrm{d} v \mathrm{~d} z=\int_{V} M(x, v, t) \mathrm{d} v=|V| M^{0}(x, t)+\mathcal{O}(\varepsilon),
$$

and if we neglect the $\mathcal{O}(\varepsilon)$ term, (4.38) leads to the following general evolution equation for $n$ :

$$
\frac{\partial n}{\partial t}=\nabla \cdot(D \nabla n-n \chi(S) \nabla S)
$$

This equation has the form of a classical chemotaxis equation (3.5), but the diffusion and the chemotactic sensitivity are tensors. It reduces to the classical chemotaxis equation, provided that the tensors (4.36) and (4.37) are isotropic, i.e., provided that $D$ and $\chi(S)$ are scalar multiples of the identity. This occurs for many turning kernels $T$; see, e.g., [32, Theorem 3.5]. For example, velocity jumps in E. coli have the property that the speed of individuals is essentially constant during the motion and the new direction is chosen randomly. For this system we consider the velocity-jump process with fixed speed $s$ but variable direction, where the turn angle distribution is constant, i.e.,

$$
V=s \mathcal{S}^{N-1}, \quad T\left(v, v^{\prime}\right)=\frac{1}{|V|},
$$

where $\mathcal{S}^{N-1}$ is the unit sphere in $\mathbb{R}^{N}$. Then we can easily evaluate all terms in (4.40). Direct calculation gives $\mathcal{B}=-I$, and, consequently, (4.36) implies that

$$
D=-\frac{1}{|V| \lambda_{0}} \int_{V} v \otimes \mathcal{B} v \mathrm{~d} v=\frac{1}{|V| \lambda_{0}} \int_{V} v \otimes v \mathrm{~d} v=\frac{s^{2}}{N \lambda_{0}} I .
$$

Similarly, one has $\left(t_{e} \lambda_{0} \mathcal{A}-I\right)^{-1}=\left(1+t_{e} \lambda_{0}\right)^{-1} I+C_{1}$ and $\left(t_{a} \lambda_{0} \mathcal{A}-I\right)^{-1}=(1+$ $\left.t_{a} \lambda_{0}\right)^{-1} I+C_{2}$, where $C_{1}$ and $C_{2}$ are independent of $v$. Consequently, (4.37) implies

$$
\begin{aligned}
\chi(S) & =\frac{b t_{a}}{|V| \lambda_{0}} g^{\prime}(S(x)) \int_{V} v \otimes\left(t_{e} \lambda_{0} \mathcal{A}-I\right)^{-1}\left(t_{a} \lambda_{0} \mathcal{A}-I\right)^{-1} v \mathrm{~d} v \\
& =\frac{b t_{a}}{|V| \lambda_{0}} g^{\prime}(S(x)) \frac{1}{1+t_{e} \lambda_{0}} \frac{1}{1+t_{a} \lambda_{0}} \int_{V} v \otimes v \mathrm{~d} v=g^{\prime}(S(x)) \frac{b s^{2} t_{a}}{N \lambda_{0}\left(1+t_{e} \lambda_{0}\right)\left(1+t_{a} \lambda_{0}\right)} .
\end{aligned}
$$

Hence, using (4.42) and (4.43) in (4.40), we derive the chemotaxis equation (3.5) for $n$, namely,

$$
\frac{\partial n}{\partial t}=\frac{s^{2}}{N \lambda_{0}} \Delta n-\nabla \cdot\left(n g^{\prime}(S(x)) \frac{b s^{2} t_{a}}{N \lambda_{0}\left(1+t_{e} \lambda_{0}\right)\left(1+t_{a} \lambda_{0}\right)} \nabla S\right) .
$$

This generalizes the derivation in [21] to an arbitrary space dimension $N$. To make the connection with the one-dimensional problem more precise, consider the transport equation (4.5) in one dimension with the turning kernel given by (4.41). Then there 
are only two velocities, $s$ and $-s$, and the right-hand side of (4.5) can be written in the form

$$
\begin{aligned}
& -\left(\lambda_{0}-b z_{1}\right) p+\int_{V}\left(\lambda_{0}-b z_{1}\right) T\left(v, v^{\prime}\right) p\left(x, v^{\prime}, z, t\right) \mathrm{d} v^{\prime} \\
= & \left(\lambda_{0}-b z_{1}\right)\left[-p(x, v, t)+\frac{1}{2}(p(x, v, t)+p(x,-v, t))\right] \\
= & \left(\frac{\lambda_{0}}{2}-\frac{b}{2} z_{1}\right)[-p(x, v, t)+p(x,-v, t)] .
\end{aligned}
$$

Consequently, we can write (4.5) in the form of one-dimensional equations (3.30) and (3.31), where the parameters $\lambda_{0}, b$ are divided by 2 . The factor of $1 / 2$ represents the probability of a jump in either direction, whereas in the earlier formulation particles were forced to reverse direction when they turned. With this in mind (4.44) is equivalent to (3.34) for $N=1$.

5. Hyperbolic models for chemotaxis. In this section we discuss another method for the derivation of macroscopic equations which is related to the method used in [21]. However, the correspondence with [21] is not complete because movement in higher dimensions introduces velocity moments that reflect the correlations between different directions. In section 4, we derived (4.13)-(4.15) for internal moments $M$, $M_{1}$, and $M_{2}$ defined by (4.6)-(4.8). For simplicity, we consider only the isotropic turning kernel given by (4.41). Then (4.13)-(4.15) read as

$$
\begin{aligned}
& \frac{\partial M}{\partial t}+\nabla \cdot v M=-\lambda_{0} M+\lambda_{0} \frac{1}{|V|} \int_{V} M\left(x, v^{\prime}, t\right) \mathrm{d} v^{\prime}+b M_{1}-b \frac{1}{|V|} \int_{V} M_{1}\left(x, v^{\prime}, t\right) \mathrm{d} v^{\prime}, \\
& (5.2) \quad \frac{\partial M_{1}}{\partial t}+\nabla \cdot v M_{1}=-\left(\lambda_{0}+\frac{1}{t_{e}}\right) M_{1}-\frac{1}{t_{e}} M_{2}+\lambda_{0} \frac{1}{|V|} \int_{V} M_{1}\left(x, v^{\prime}, t\right) \mathrm{d} v^{\prime},
\end{aligned}
$$

$$
\frac{\partial M_{2}}{\partial t}+\nabla \cdot v M_{2}=-g^{\prime}(S(x)) \nabla S \cdot v M-\left(\lambda_{0}+\frac{1}{t_{a}}\right) M_{2}+\lambda_{0} \frac{1}{|V|} \int_{V} M_{2}\left(x, v^{\prime}, t\right) \mathrm{d} v^{\prime} .
$$

To analyze these we define the following moments:

$$
\begin{aligned}
& n(x, t)=\int_{V} \int_{\mathbb{R}^{2}} p(x, v, z, t) \mathrm{d} z \mathrm{~d} v=\int_{V} M(x, v, t) \mathrm{d} v, \\
& n_{i}(x, t)=\int_{V} \int_{\mathbb{R}^{2}} z_{i} p(x, v, z, t) \mathrm{d} z \mathrm{~d} v=\int_{V} M_{i}(x, v, t) \mathrm{d} v, \quad i \in\{1,2\}, \\
& j^{k}(x, t)=\int_{V} \int_{\mathbb{R}^{2}} v_{k} p(x, v, z, t) \mathrm{d} z \mathrm{~d} v=\int_{V} v_{k} M(x, v, t) \mathrm{d} v, \quad k \in\{1, \ldots, N\}, \\
& j_{i}^{k}(x, t)=\int_{V} \int_{\mathbb{R}^{2}} v_{k} z_{i} p(x, v, z, t) \mathrm{d} z \mathrm{~d} v=\int_{V} v_{k} M_{i}(x, v, t) \mathrm{d} v, \quad k \in\{1, \ldots, N\}, \\
& i \in\{1,2\},
\end{aligned}
$$

$$
j^{k l}(x, t)=\int_{V} \int_{\mathbb{R}^{2}} v_{k} v_{l} p(x, v, z, t) \mathrm{d} z \mathrm{~d} v=\int_{V} v_{k} v_{l} M(x, v, t) \mathrm{d} v, \quad k, l \in\{1, \ldots, N\},
$$




$$
\begin{gathered}
j_{i}^{k l}(x, t)=\int_{V} \int_{\mathbb{R}^{2}} v_{k} v_{l} z_{i} p(x, v, z, t) \mathrm{d} z \mathrm{~d} v=\int_{V} v_{k} v_{l} M_{i}(x, v, t) \mathrm{d} v, \quad k, l \in\{1, \ldots, N\}, \\
i \in\{1,2\} .
\end{gathered}
$$

Here, $n(x, t)$ is the particle density, $n_{1}(x, t)$ and $n_{2}(x, t)$ are moments with respect to the internal variables, and $j^{k}(x, t), k=1, \ldots, N$, are fluxes. Multiplying (5.1)-(5.3) by $1, v_{1}, v_{2}, \ldots, v_{N}$, respectively, and integrating the resulting equation with respect to $v$, we obtain the following $3(N+1)$ moment equations:

$$
\begin{gathered}
\frac{\partial n}{\partial t}+\sum_{k=1}^{N} \frac{\partial j^{k}}{\partial x_{k}}=0, \\
\frac{\partial j^{k}}{\partial t}+\sum_{l=1}^{N} \frac{\partial j^{k l}}{\partial x_{l}}=-\lambda_{0} j^{k}+b j_{1}^{k}, \quad k=1,2, \ldots, N, \\
\frac{\partial n_{1}}{\partial t}+\sum_{k=1}^{N} \frac{\partial j_{1}^{k}}{\partial x_{k}}=-\frac{1}{t_{e}} n_{1}-\frac{1}{t_{e}} n_{2}, \\
\frac{\partial j_{1}^{k}}{\partial t}+\sum_{l=1}^{N} \frac{\partial j_{1}^{k l}}{\partial x_{l}}=-\left(\lambda_{0}+\frac{1}{t_{e}}\right) j_{1}^{k}-\frac{1}{t_{e}} j_{2}^{k}, \quad c=1,2, \ldots, N, \\
\frac{\partial n_{2}}{\partial t}+\sum_{k=1}^{N} \frac{\partial j_{2}^{k}}{\partial x_{k}}=-\frac{1}{t_{a}} n_{2}-g^{\prime}(S(x)) \nabla S \cdot\left(\begin{array}{c}
j^{1} \\
j^{2} \\
\vdots \\
j^{N}
\end{array}\right),
\end{gathered}
$$

$$
\frac{\partial j_{2}^{k}}{\partial t}+\sum_{l=1}^{N} \frac{\partial j_{2}^{k l}}{\partial x_{l}}=-\left(\lambda_{0}+\frac{1}{t_{a}}\right) j_{2}^{k}-g^{\prime}(S(x)) \nabla S \cdot\left(\begin{array}{c}
j^{k 1} \\
j^{k 2} \\
\vdots \\
j^{k N}
\end{array}\right), \quad k=1,2, \ldots, N
$$

This system of $3(N+1)$ equations for $3(N+1)$ moments $n, n_{i}, j^{k}, j_{i}^{k}, i=1,2$, $k=1,2, \ldots, N$, also contains higher-order (internal-)velocity moments $j^{k l}, j_{i}^{k l}, k, l \in$ $\{1, \ldots, N\}, i=1,2$. To close this system we choose the moment closure

$$
j^{k l}=\frac{s^{2}}{N} n \delta_{k l}, \quad j_{i}^{k l}=\frac{s^{2}}{N} n_{i} \delta_{k l} \quad \text { for } k, l \in\{1, \ldots, N\}, i \in\{1,2\},
$$

and then (5.10)-(5.15) take the form

$$
\begin{gathered}
\frac{\partial n}{\partial t}+\sum_{k=1}^{N} \frac{\partial j^{k}}{\partial x_{k}}=0, \\
\frac{\partial j^{k}}{\partial t}+\frac{s^{2}}{N} \frac{\partial n}{\partial x_{k}}=-\lambda_{0} j^{k}+b j_{1}^{k}, \quad k=1,2, \ldots, N,
\end{gathered}
$$




$$
\begin{gathered}
\frac{\partial n_{1}}{\partial t}+\sum_{k=1}^{N} \frac{\partial j_{1}^{k}}{\partial x_{k}}=-\frac{1}{t_{e}} n_{1}-\frac{1}{t_{e}} n_{2}, \\
\frac{\partial j_{1}^{k}}{\partial t}+\frac{s^{2}}{N} \frac{\partial n_{1}}{\partial x_{k}}=-\left(\lambda_{0}+\frac{1}{t_{e}}\right) j_{1}^{k}-\frac{1}{t_{e}} j_{2}^{k}, \quad k=1,2, \ldots, N, \\
\frac{\partial n_{2}}{\partial t}+\sum_{k=1}^{N} \frac{\partial j_{2}^{k}}{\partial x_{k}}=-\frac{1}{t_{a}} n_{2}-g^{\prime}(S(x)) \nabla S \cdot\left(\begin{array}{c}
j^{1} \\
j^{2} \\
\vdots \\
j^{N}
\end{array}\right), \\
\frac{\partial j_{2}^{k}}{\partial t}+\frac{s^{2}}{N} \frac{\partial n_{2}}{\partial x_{k}}=-\left(\lambda_{0}+\frac{1}{t_{a}}\right) j_{2}^{k}-\frac{s^{2}}{N} g^{\prime}(S(x)) \frac{\partial S}{\partial x_{k}} n, \quad k=1,2, \ldots, N .
\end{gathered}
$$

This system is closed, and one can follow the analysis from [21] to derive (on a time scale $\mathcal{O}(1)$ sec and a space scale $\mathcal{O}(1-10) \mathrm{mm}$ ) a hyperbolic version of the classical chemotaxis equation

$$
\frac{1}{\lambda_{0}} \frac{\partial^{2} n}{\partial t^{2}}+\frac{\partial n}{\partial t}=\frac{s^{2}}{N \lambda_{0}} \triangle n-\nabla \cdot\left(g^{\prime}(S(x)) \frac{b s^{2} t_{a}}{N \lambda_{0}\left(1+t_{e} \lambda_{0}\right)\left(1+t_{a} \lambda_{0}\right)} \nabla S n\right) .
$$

In particular, (5.23) is equivalent to (3.33) if $\lambda_{0}$ and $b$ are interpreted according to (4.45) and according to comments after (4.45). Moreover, the asymptotic behavior of the damped wave equation (5.23) is given by (4.44), which suggests that the moment closure (5.16) is appropriate. This closure is used in [31] for systems without internal dynamics, and some estimates of the error are given there.

6. An illustrative numerical example. The macroscopic descriptions of chemotaxis embodied in either the modified classical chemotaxis equation (5.23) or the classical chemotaxis equation (4.44) are approximations to the original transport equation and the stochastic process describing movement that underlies it. The computational results for one dimension reported in [21] show that the macroscopic equations capture the behavior of the microscopic movement process very well, and here we show the results for a multidimensional example.

We suppose that all parameters are dimensionless, we choose $b=1, t_{e}=0, t_{a}=1$, $\lambda_{0}=1$, and $g=$ Identity, and we use the turning kernel given by (4.41) with $s=0.1$. This incorporates the fact that $s$ is small on the relevant macroscopic time and space scales. We consider the motion of the individuals in the rectangle $\Omega=[0,20] \times[0,10]$ with the stationary continuous piecewise linear signal $S(x)$ given as follows: $S(x)$ is 0 on the boundaries of rectangle $\Omega, S(x)$ is piecewise linear with maximum at the point $(12,6)$, and $S(12,6)=8$. We suppose that all cells are initially at the point $(10,3)$, i.e., that $n(x, 0)$ is proportional to Dirac delta function $\delta_{(10,3)}$. Moreover, we assume that all individuals are perfectly adapted at $t=0$, and we use no-flux boundary conditions.

To simulate the random walk of individuals, we consider an ensemble of $10^{6}$ particles. Each particle is described by its position $\left(x_{1}, x_{2}\right)$, its internal state $y$, and its velocity $s(\cos (\theta), \sin (\theta))$, where $\theta$ is the direction of travel. We use a small time step $d t=0.01$ (i.e., the unbiased turning frequency divided by 100). During each time step the particle moves with speed $s$ in the chosen direction $\theta$, and we integrate the 

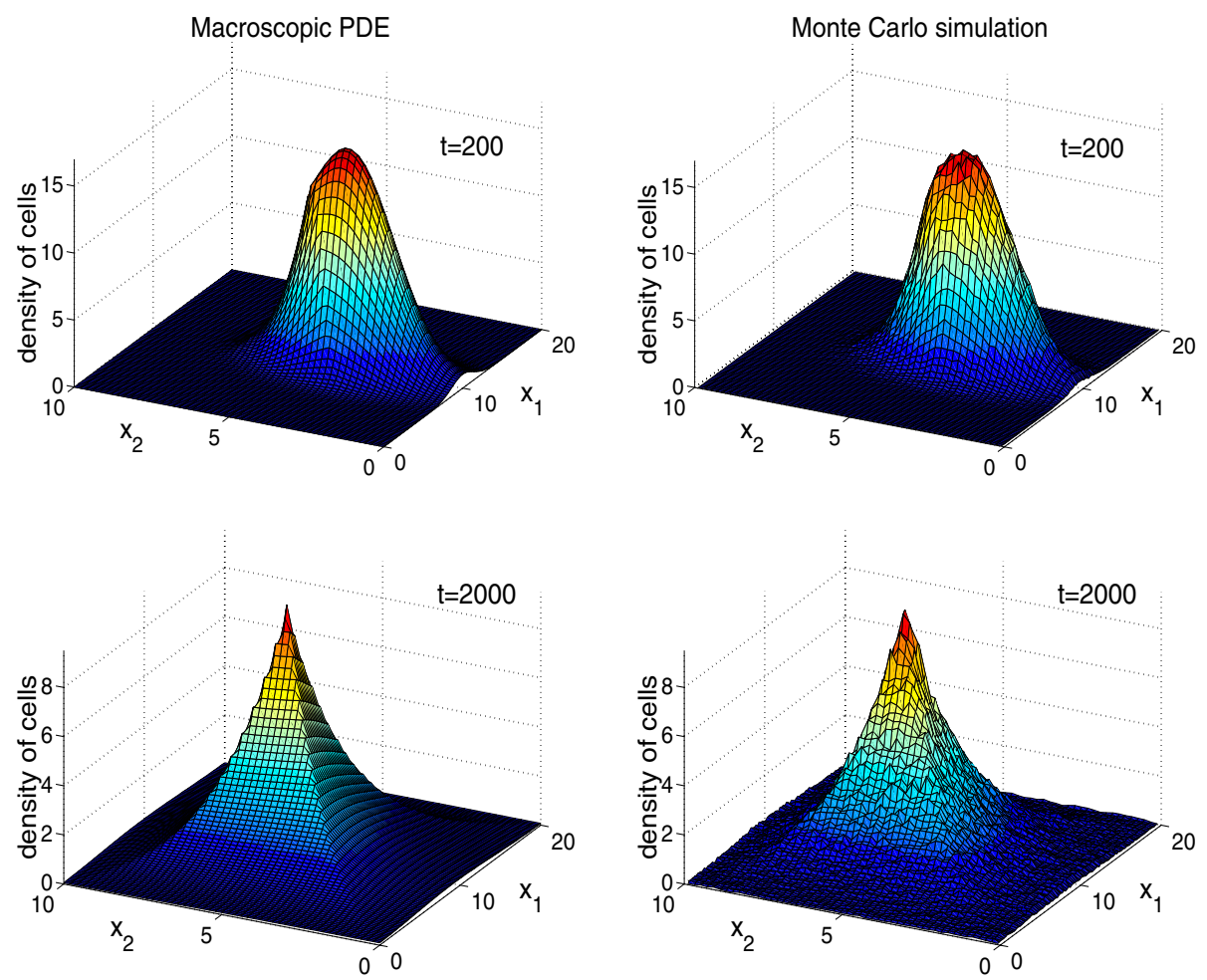

FIG. 6.1. The time evolution of the density of individuals for times $T=200$ (upper) and $T=$ 2000 (lower). The solution of (4.44) is on the left, and the stochastic simulation with $10^{6}$ individuals is shown on the right. The values of parameters, the initial condition, and the underlying signal are given in the text.

internal dynamics to find the change of $y$. At the end of each time step, a random number from $[0,1]$ is generated and compared with the probability of the turn $\lambda(y) \mathrm{d} t$. If the turn occurs, a random angle $\theta \in[0,2 \pi)$ is generated, and during the next time step the bacterium moves with the new velocity $s(\cos (\theta), \sin (\theta))$.

In Figure 6.1 we compare the results of the stochastic simulation of the random walk with the solutions of the classical chemotaxis equation (4.44). We see that the macroscopic equation gives quantitatively similar results to the stochastic simulations.

7. Discussion. Our purpose here was to derive the macroscopic chemotaxis equation for the evolution of a population by incorporating internal dynamics for the detection and processing of, and response to, external signals. This led to (4.40), (4.44) and (5.23) but required that we use sufficiently simple descriptions of the internal dynamics that capture the essential characteristics of microscopic behavior. In particular, we were able to show how characteristics of the signal transduction and behavioral response are reflected in the chemotactic sensitivity. The motivating problem was the behavior of flagellated bacteria, but the mathematical approach presented here can be applied to any biological system, provided that we appropriately describe the signal transduction and response. Of course the entire analysis is based on the premise that the transport equation describes the microscopic process, and this is known to be true for noninteracting walkers in a fixed signal field. Whether the same equation still holds when the walkers interact directly or produce and degrade a 
diffusible signal is an open question. Other restrictions used in the analysis are that the signal gradient be small in an appropriate sense and that the internal dynamics be close to equilibrium. The limitations of the former remain to be determined, but the limitation of the latter may not be so important unless adaptation is slow compared to the time scale of movement.

The derivation of macroscopic evolution equations for the density becomes more difficult if one attempts to incorporate more details of signal transduction and the behavioral response. Even if one assumes that the internal dynamics are close to equilibrium so that a linearized response can be used, it is still difficult to extract the slow linear modes and the dependence of the response on the signal from the nonlinear evolution equations. As we indicated earlier, the signal transduction network in $E$. coli is very complicated, but the input-output behavior is very simple, and, as we showed in [21], the cartoon model reflects the dynamics very well. In general this will not be true, and methods for systematically incorporating complex internal dynamics are needed.

Another difficulty arises in dense aggregates, in which the walkers interact directly, or perhaps indirectly, via the signal field. Chemotaxis equations for indirectly interacting particles have been derived by Stevens [65], who shows that the chemotaxis system can be derived in suitable limits. That analysis is based on the assumption that the microscopic velocity of cells is proportional to the gradient of a smoothed density of the attractant. This assumes that cells measure the gradient and speed up in proportion to its magnitude, but, as we described earlier, this is the case neither for bacteria nor for many amoeboid cells. A different approach in which finite-volume effects are included formally is given in [50]. The derivation of continuum limits of interacting particle systems based on a more realistic description of how cells sample the environment and process the signals involves significant technical difficulties and has not been done at present. Harder still is the problem in which the cells interact directly via receptors on their membranes or, in the bacterial case, via interaction of their flagella. Here it is not even known whether continuum limits exist in general, but heuristics-based continuum models for bacterial populations have been used very effectively [41].

The technical difficulties involved in rigorous derivation of continuum limits suggest that alternate computational approaches may be useful, and in the remainder of this section we describe such methods. In the case of bacterial pattern formation (see section 2.3), a naive approach would be to simulate the random walk of bacteria using direct Monte Carlo simulation, but there are several obstacles to doing this. First, the number of bacteria is very large: there are approximately $50 \mathrm{~K}$ bacteria initially, they divide every 2 hours, and the time scale of interest is days. Second, the present models of signal transduction can involve 20 or more ODEs, and these equations have to be solved along the trajectory of each bacterium. Consequently, the direct Monte Carlo simulation of the experimental setup is currently not possible on the time scale of interest. Thus there is a need to develop new computational techniques, and the bacterial colony patterns can be viewed as a test problem of the predictive power of any method for lifting cell-level behavior to macroscopic, population-level descriptions.

Let us begin with a simple example to illustrate the ideas behind a new approach [39] (one method was already applied to bacterial chemotaxis in [60]). Suppose that the evolution of the density of individuals $n$ is given by the known PDE

$$
\frac{\partial n}{\partial t}=\mathcal{L}(n),
$$




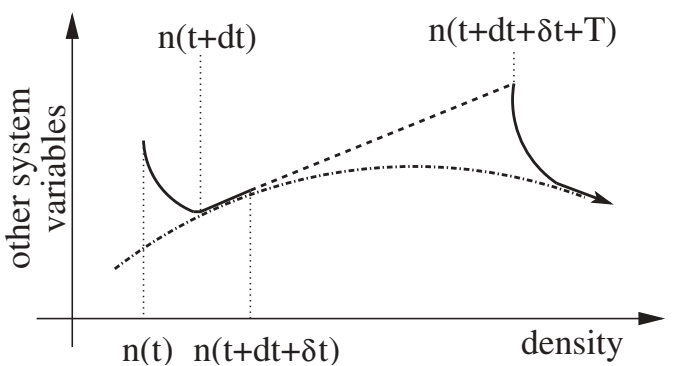

(a)

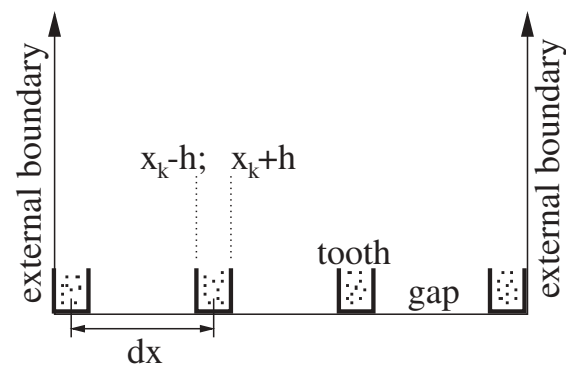

(b)

FIG. 7.1. (a) Schematic illustration of the coarse integration method. First we run the full microscopic model for a short time $d t$, which will allow the dynamics to approach a "slow manifold" (dotted-dashed line) parametrized by density. Then we evolve the system on the slow manifold for another short time $\delta t$ and use the information about density in the interval $[t+d t, t+d t+\delta t]$ to find the density at time $t+d t+\delta t+T$ by an appropriate extrapolation. (b) Schematic illustration of the gap-tooth scheme. We choose a macroscopic space step $d x$ and macroscopic meshpoints $x_{k}$ that are dx apart. To determine the values of the density $n\left(x_{k}\right)$ at meshpoints, we construct a small domain (a "tooth") around each meshpoint, and we run the stochastic simulation only in the teeth with suitably chosen boundary conditions.

where $\mathcal{L}$ is an operator on a suitable Banach space, and suppose we are interested in the steady states of the system. A naive approach would be to use a PDE solver for (7.1) and compute the solution for times $t$ large enough that further changes in time are acceptably small. However, in the case of PDEs, we can also use what is usually a faster approach - we can use a Newton-Raphson-type of iterative method to solve the steady state equation $\mathcal{L}(n)=0$. Of course either approach is impossible without $\mathcal{L}(n)$, but in such cases we assume that the microscopic model of the dynamics provides a time stepper - a method to compute the state of the system at time $t+\mathrm{d} t$ from the state of the system at time $t$. This can then be used together with a Newton-GMRES iteration for finding the steady states and doing bifurcation analysis in the absence of $\mathcal{L}(n)$ (see, e.g., $[42,39]$ and the references therein).

If we are interested in the slow dynamics of the system, one way to speed up the direct simulations is to use what is called the coarse integration method. The idea of coarse integration is sketched in Figure 7.1(a). It is based on explicitly assuming that there is a separation of time scales such that higher-order moments of a distribution relax quickly to functionals of lower-order moments; in particular, in the bacterial case we assume that the system is described in terms of density only on a long time scale. The analysis in earlier sections shows when this is appropriate under the conditions used to derive the chemotaxis equation. The simplest computational algorithm is as follows. First we run the full Monte Carlo simulation for a short time $\mathrm{d} t$ (see Figure 7.1(a)), which will allow us to compute the density of individuals $n(t+\mathrm{d} t)$ from the density of individuals $n(t)$. This will allow the fast processes to equilibrate, i.e., to approach a "slow manifold" parametrized by the density $n$. Next, we run the dynamics on the slow manifold for another short time interval $\delta t$ to find density $n(t+\mathrm{d} t+\delta t)$. Finally, we use simple extrapolation based on the values of $n$ in the interval $[t+\mathrm{d} t, t+\mathrm{d} t+\delta t]$ to find the value of the solution at time $t+\mathrm{d} t+\delta t+T$, where $T$ is a macroscopic time. Theoretical justification of the projective integration was done in the case of ODEs with a gap in the spectrum in [25], and the method was applied with a Monte Carlo simulation of bacterial chemotaxis in [60]. Clearly, the method can speed up the computations, provided that $T \gg \mathrm{d} t+\delta t$. 
One can also reduce the simulations from large spatial domains to small ones using the gap-tooth scheme $[26,56]$. The idea is the following. Consider the onedimensional problem as it is schematically drawn in Figure 7.1(b). We choose a macroscopic space step $\mathrm{d} x$ and macroscopic meshpoints $x_{k}$ with distance $\mathrm{d} x$ apart. We are interested in the values of the density of particles $n\left(x_{k}\right)$ at meshpoints, and to compute them we construct the small domain (a tooth) around each meshpoint defined by the interval $\left[x_{k}-h, x_{k}+h\right]$ and run the stochastic simulation only in teeth. Clearly, the method can speed up the computations, provided that $\mathrm{d} x \gg h$ and that we can introduce good boundary conditions for each tooth (see, e.g., $[26,56]$ and the references therein). Finally, we can also combine the gap-tooth scheme with coarse integration to connect "small space, short time" simulations with "large space, long time" evolution. This combination is called patch dynamics [39].

To summarize this discussion, if the direct simulations are too slow to obtain results in a reasonable amount of time, one has to build some computational superstructure around the Monte Carlo simulator to answer macroscopic questions computationally using microscopic models. The advantages and disadvantages of the computational methods presented above require further investigation, and this is ongoing research.

\section{REFERENCES}

[1] R. Albert, Y. Chiu, and H. G. Othmer, Dynamic receptor team formation can explain the high signal transduction gain in E. coli, Biophys. J., 86 (2004), pp. 2650-2659.

[2] M. J. Ashby, J. E. Neale, S. J. Knott, And I. A. CRitchley, Effect of antibiotics on non-growing planktonic cells and biofilms of escherichia coli, Journal of Antimicrobial Chemotherapy, 33 (1994), pp. 443-452.

[3] N. Barkai And S. Leibler, Robustness in simple biochemical networks, Nature (London), 387 (1997), pp. 913-917.

[4] E. Ben-Jacob, I. Cohen, O. Shochet, I. Aranson, and H. Levine, Complex bacterial patterns, Nature, 373 (1995), pp. 566-567.

[5] E. Ben-Jacob, O. Schochet, A. Tenenbaum, I. Cohen, and A. Czirok, Generic modelling of cooperative growth patterns in bacterial colonies, Nature, 368 (1994), pp. 46-49.

[6] H. C. Berg, A physicist looks at bacterial chemotaxis, Cold Spring Harbor Symposia on Quantitative Biology, 53 (1988).

[7] H. C. Berg and E. M. Purcell, Physics of chemoreception, Biophys. J., 20 (1977), pp. 193-219.

[8] S. Block, J. Segall, and H. Berg, Adaptation kinetics in bacterial chemotactics, J. Bacteriol., 154 (1983), pp. 312-323.

[9] S. M. Block, J. E. Segall, and H. C. Berg, Impulse responses in bacterial chemotaxis, Cell, 31 (1982), pp. 215-226.

[10] R. B. Bourret, K. A. Borkovich, And M. I. Simon, Signal transduction pathways involving protein phosphorylation in prokaryotes, Ann. Rev. Biochem., 60 (1991), pp. 401-441.

[11] A. Boyd AND M. I. Simon, Multiple electrophoretic forms of methyl-accepting chemotaxis proteins generated by stimulus-elicited methylation in Escherichia coli, J. Bacteriol., 143 (1980), pp. 809-815.

[12] D. Bray And R. B. Bourret, Computer analysis of the binding reactions leading to a transmembrane receptor-linked multiprotein complex involved in bacterial chemotaxis, Mol. Biol. of the Cell, 6 (1995), pp. 1367-1380.

[13] M. P. Brenner, L. S. Levitov, and E. O. Budrene, Physical mechanisms for chemotactic pattern formation by bacteria, Biophys. J., 74 (1998), pp. 1677-1693.

[14] E. BUDRENE, private communication, 2003.

[15] E. Budrene And H. Berg, Complex patterns formed by motile cells of Escherichia coli, Nature, 349 (1991), pp. 630-633.

[16] E. O. BUdRene AND H. Berg, Dynamics of formation of symmetrical patterns by chemotactic bacteria, Nature, 376 (1995), pp. 49-53.

[17] S. R. Caplan And M. Eisenbach, Bacterial chemotaxis: Unsolved mystery of the flagellar switch, Curr. Biol., 8 (1998), pp. R444-R446. 
[18] P. Cluzel, M. Surette, And S. Leibler, An ultrasensitive bacterial motor revealed by monitoring signaling proteins in single cells, Science, 287 (2000), pp. 1652-1655.

[19] J. W. Costerton, Z. Lewandowski, D. E. Caldwell, D. R. Korber, and H. M. LappinSсотт, Microbial biofilms, Ann. Rev. Microbiol., 49 (1995), pp. 711-745.

[20] Y. Dolak and C. Schmeiser, Kinetic Models for Chemotaxis: Hydrodynamic Limits and the Back-of-the-Wave Problem, ANUM preprint, Vienna University of Technology, Vienna, Austria, 2003.

[21] R. Erban and H. G. Отнmer, From individual to collective behavior in bacterial chemotaxis, SIAM J. Appl. Math., 65 (2004), pp. 361-391.

[22] J. J. Falke and G. L. Hazelbauer, Transmembrane signaling in bacterial chemoreceptors, Trends Biochem. Sci., 26 (2001), pp. 257-265.

[23] A. Filloux and I. Vallet, Biofilm: Set-up and organization of a bacterial community, Med. Sci. (Paris), 19 (2003), pp. 77-83.

[24] R. FürTh, Die Brownsche Bewegung bei Berücksichtigung einer Persistenz der Bewegungsrichtung, Zeitsch. f. Physik, 2 (1920), pp. 244-256.

[25] C. W. Gear and I. G. KevreKidis, Projective methods for stiff differential equations: Problems with gaps in their eigenvalue spectrum, SIAM J. Sci. Comput., 24 (2003), pp. 1091-1105.

[26] C. Gear, J. Li, and I. Kevrekidis, The gap-tooth method in particle simulations, Phys. Lett. A, 316 (2003), pp. 190-195.

[27] J. A. Gegner, D. R. Graham, A. F. Roth, and F. W. Dahlquist, Assembly of an MCP receptor, Che $W$ and kinase CheA complex in the bacterial chemotaxis signal transduction pathway, Cell, 70 (1992), pp. 975-982.

[28] S. Goldstein, On diffusion by discontinuous movements, and on the telegraph equation, Quart. J. Mech. Appl. Math., 6 (1951), pp. 129-156.

[29] G. J. HabetLer and MatKowsky, Uniform asymptotic expansion in transport theory with small mean free paths, and the diffusion approximation, J. Mathematical Phys., 4 (1975), pp. $846-854$.

[30] C. Helmstetter, Timing of synthetic activities in the cell cycle, in Escherichia coli and Salmonella: Cellular and Molecular Biology, F. Neidhardt, ed., ASM Press, Washington, DC, 1996, pp. 1627-1639.

[31] T. Hillen, Transport Equations and Chemosensitive Movement, habilitation thesis, University of Tuebingen, Germany, 2001.

[32] T. Hillen and H. G. Othmer, The diffusion limit of transport equations derived from velocityjump processes, SIAM J. Appl. Math., 61 (2000), pp. 751-775.

[33] T. Hillen and A. Stevens, Hyperbolic models for chemotaxis in 1-D, Nonlinear Anal. Real World Appl., 1 (2000), pp. 409-433.

[34] D. Horstmann, K. Painter, and H. G. Othmer, Aggregation under local reinforcement: From lattice to continuum, European J. Appl. Math., to appear.

[35] M. KAC, A stochastic model related to the telegrapher's equation, Rocky Mountain J. Math., 3 (1974), pp. 497-509.

[36] K. Kawasaki, A. Mochizuki, M. Matsushita, T. Umeda, and N. Shigesada, Modeling spatiotemporal patterns generated by Bacillus subtilis, J. Theoret. Biol., 188 (1997), pp. 177-185.

[37] E. Keller and L. Segel, Model for chemotaxis, J. Theoret. Biol., 30 (1971), pp. 225-234.

[38] E. Keller and L. Segel, Traveling bands of chemotactic bacteria: A theoretical analysis, J. Theoret. Biol., 30 (1971), pp. 235-248.

[39] I. Kevrekidis, C. Gear, J. Hyman, P. Kevrekidis, O. Runborg, and C. Theodoropoulos, Equation-free, coarse-grained multiscale computation: Enabling microscopic simulators to perform system-level analysis, Commun. Math. Sci., 1 (2003), pp. 715-762.

[40] E. W. Larsen AND J. B. Keller, Asymptotic solution of neutron transport problems for small free mean paths, J. Mathematical Phys., 15 (1974), pp. 75-81.

[41] J. Lega And T. Passot, Hydrodynamics of bacterial colonies: A model, Phys. Rev. E (3), 67 (2003), 031906.

[42] A. Makeev, D. Maroudas, and I. Kevrekidis, "Coarse" stability and bifurcation analysis using stochastic simulators: Kinetic Monte Carlo examples, J. Chem. Phys., 116 (2002), pp. 10083-10091.

[43] H. MCKean, Chapman-Enskog-Hilbert expansions for a class of solutions of the telegraph equation, J. Mathematical Phys., 8 (1967), pp. 547-552.

[44] N. Mittal, E. O. Budrene, M. P. Brenner, and A. van Oudenaarden, Motility of escherichia coli cells in clusters formed by chemotactic aggregation, Proc. Natl. Acad. Sci. USA, 100 (2003), pp. 13259-13263.

[45] C. J. Morton-Firth, T. S. Shimizu, and D. Bray, A free-energy-based stochastic simulation of the tar receptor complex, J. Mol. Biol., 286 (1999), pp. 1059-1074. 
[46] H. Othmer, S. Dunbar, and W. Alt, Models of dispersal in biological systems, J. Math. Biol., 26 (1988), pp. 263-298.

[47] H. Othmer And P. SchaAp, Oscillatory cAMP signaling in the development of Dictyostelium discoideum, Comments on Theoretical Biology, 5 (1998), pp. 175-282.

[48] H. G. Отhmer And A. Stevens, Aggregation, blowup, and collapse: The ABC's of taxis in reinforced random walks, SIAM J. Appl. Math., 57 (1997), pp. 1044-1081.

[49] H. G. Othmer and T. Hillen, The diffusion limit of transport equations II: Chemotaxis equations, SIAM J. Appl. Math., 62 (2002), pp. 1222-1250.

[50] K. J. Painter and T. Hillen, Volume-filling and quorum sensing in models for chemosensitive movement, Can. Appl. Math. Q., 10 (2002), pp. 501-543.

[51] K. J. Painter, D. Horstmann, and H. G. Othmer, Localization in lattice and continuum models of reinforced random walks, Appl. Math. Lett., 16 (2003), pp. 375-381.

[52] K. J. Painter, P. K. Maini, And H. G. Othmer, Development and applications of a model for cellular response to multiple chemotactic cues, J. Math. Biol., 41 (2000), pp. 285-314.

[53] G. C. Papanicolaou, Asymptotic analysis of transport processes, Bull. Amer. Math. Soc., 81 (1975), pp. 330-392.

[54] M. Pasmore And J. W. Costerton, Biofilms, bacterial signaling, and their ties to marine biology, J. Ind. Microbiol. Biotechnol., 30 (2003), pp. 407-413.

[55] E. M. Purcell, Life at low Reynolds number, Amer. J. Phys., 45 (1977), pp. 3-11.

[56] G. Samaey, D. Roose, and I. G. KevreKidis, The gap-tooth scheme for homogenization problems, Multiscale Model. Simul., to appear.

[57] K. SAuer, The genomics and proteomics of biofilm formation, Genome Biol., 4 (2003), p. 219.

[58] J. E. Segall, S. M. Block, And H. C. Berg, Temporal comparisons in bacterial chemotaxis, Proc. Nat. Acad. Sci. U.S.A., 83 (1986), pp. 8987-8991.

[59] L. A. Segel, Mathematical models for cellular behavior, in Studies in Mathematical Biology, S. A. Levin, ed., MAA, Washington, DC, 1978, pp. 156-190.

[60] S. Setayeshgar, C. W. Gear, H. G. Othmer, and I. G. Kevrekidis, Application of coarse integration to bacterial chemotaxis, Multiscale Model. Simul., to appear.

[61] J. A. ShAPIRO, Thinking about bacterial populations as multicellular organisms, Ann. Rev. Microbiol., 52 (1998), pp. 81-104.

[62] S. A. Simms, A. M. Stock, And J. B. Stock, Purification and characterization of the S-adenosylmethionine:glutamyl methyltransferase that modifies membrane chemoreceptor proteins in bacteria, J. Biol. Chem., 262 (1987), pp. 8537-8543.

[63] V. Sourjik And H. C. Berg, Receptor sensitivity in bacterial chemotaxis, Proc. Natl. Acad. Sci. USA, 99 (2002), pp. 123-127.

[64] P. Spiro, J. Parkinson, and H. Othmer, A model of excitation and adaptation in bacterial chemotaxis, Proc. Nat. Acad. Sci. U.S.A., 94 (1997), pp. 7263-7268.

[65] A. Stevens, The derivation of chemotaxis equations as limit dynamics of moderately interacting stochastic many-particle systems, SIAM J. Appl. Math., 61 (2000), pp. 183-212.

[66] R. C. Stewart and F. W. Dahlquist, Molecular components of bacterial chemotaxis, Chem. Rev., 87 (1987), pp. 997-1025.

[67] J. B. Sтоск, Adaptative responses in bacterial chemotaxis, in Regulation of Cellular Signal Transduction Pathways by Desensitization and Amplification, D. R. Sibley and M. D. Houslay, eds., Wiley and Sons, New York, London, Sydney, 1994, pp. 3-24.

[68] J. B. Stock and M. G. Surette, Chemotaxis, in Escherichia coli and Salmonella: Cellular and Molecular Biology, Vol. I, F. Neidhardt, ed., ASM Press, Washington, DC, 1996, pp. $1103-1129$.

[69] D. W. Stroock, Some stochastic processes which arise from a model of the motion of a bacterium, Probab. Theory Related Fields, 28 (1974), pp. 305-315.

[70] G. I. TAYlor, Diffusion by continuous movements, Proc. Lon. Math. Soc., 20 (1920), pp. $196-212$.

[71] T. C. Terwilliger, J. Y. Wang, and D. E. Koshland, Kinetics of receptor modification, J. Biol. Chem., 261 (1986), pp. 10814-10820.

[72] L. Tsimring, H. Levine, I. Aranson, E. Ben-Jacob, I. Cohen, O. Shochet, and W. N. REYNoldS, Aggregation patterns in stressed bacteria, Phys. Rev. Lett., 75 (1995), pp. 18591862.

[73] D. E. Woodward, R. Tyson, M. R. Myerscough, J. D. Murray, E. O. Budrene, and H. C. Berg, Spatio-temporal patterns generated by salmonella typhimurium, Biophys. J., 68 (1995), pp. 2181-2189.

[74] E. Zauderer, Partial Differential Equations of Applied Mathematics, Wiley, New York, 1983. 